Hydrol. Earth Syst. Sci. Discuss., 8, 917-955, 2011

www.hydrol-earth-syst-sci-discuss.net/8/917/2011/

doi:10.5194/hessd-8-917-2011

(C) Author(s) 2011. CC Attribution 3.0 License.

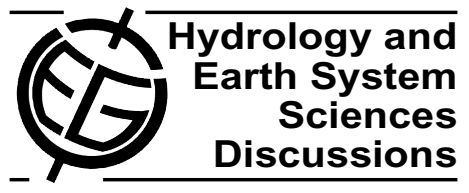

HESSD

8, 917-955, 2011

This discussion paper is/has been under review for the journal Hydrology and Earth System Sciences (HESS). Please refer to the corresponding final paper in HESS if available.

\title{
Comparison of predictions of rainfall-runoff models for changes in rainfall in the Murray-Darling Basin
}

\section{J. M. Whyte ${ }^{1}$, A. Plumridge ${ }^{2}$, and A. V. Metcalfe ${ }^{1}$}

${ }^{1}$ School of Mathematical Sciences, The University of Adelaide, Australia

${ }^{2}$ School of Mathematics and Statistics, The University of South Australia, Australia

Received: 24 December 2010 - Accepted: 5 January 2011 - Published: 24 January 2011

Correspondence to: J. M. Whyte (jason.whyte@adelaide.edu.au)

Published by Copernicus Publications on behalf of the European Geosciences Union.
Comparison of rainfall-runoff models in the Murray-Darling Basin

J. M. Whyte et al.

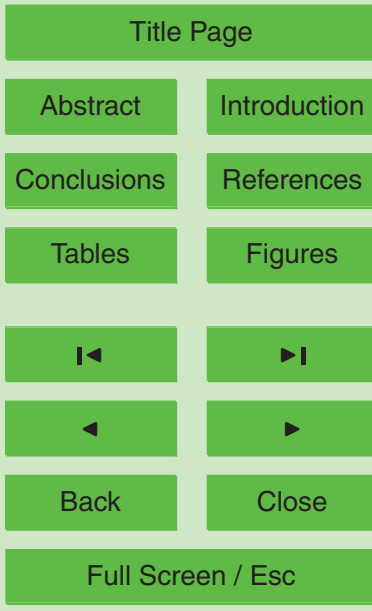

Printer-friendly Version

Interactive Discussion 


\section{Abstract}

Management of water resources requires an appreciation for how climate change, in particular changes in rainfall, affects the volume of water available in runoff. While there are many studies that use hydrological models for this purpose, comparisons of 5 predictions appear much less commonly in the literature. This paper aims to contribute to this discussion by proposing methods for evaluating the effect on daily runoff projections of rainfall-runoff models when historical daily rainfall inputs are scaled by factors that increase and decrease the rainfall. Considered are the widely used lumped conceptual model SIMHYD and a selection of time series models which feature lagged 10 runoff and rainfall terms. In particular these are AutoRegressive with eXogenous input (ARX), a variant containing nonlinear autoregressive runoff terms (NARX), a model for the log transform of runoff, a finite impulse response model (FIR) and a two regime threshold autoregressive model with exogenous input (TARX).

Results show that SIMHYD and the single regime time series models considered 15 have very different behaviour under scaled input rainfall. Reasons for the discrepancy are discussed. The amplification of the rainfall change observed for SIMHYD is consistent with claims that a $1 \%$ change in rainfall leads to a $2-3 \%$ change in runoff in the Murray-Darling Basin.

\section{Introduction}

20 Recent reductions in river flows (runoff) in Australia's Murray-Darling basin (MDB), together with an increasing population, have resulted in extended periods when there is insufficient water to satisfy demand. The consequential economic and ecological costs have intensified interest in management of the MDB. One aspect of this is an understanding of the relationship between rainfall and runoff as this informs estimates of water availability used subsequently in determining water allocations.

Modelling future runoff in an Australian context is subject to considerable uncertainty as changes to historically observed rainfall levels are an expected feature of climate
HESSD

8, 917-955, 2011

Comparison of rainfall-runoff models in the Murray-Darling Basin

J. M. Whyte et al.

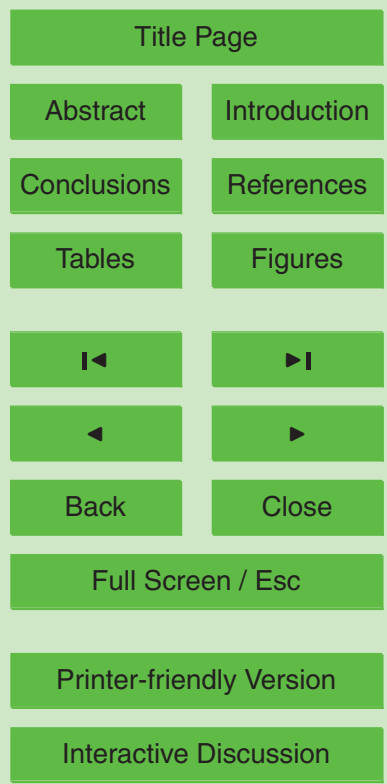


change. The rainfall section of a recent CSIRO and Australian Bureau of Meteorology report summary (CSIRO and the Australian Bureau of Meteorology, 2007, Pages 5-6) (giving a selection of results from a report (CSIRO and ABS, 2007)) gives projections for rainfall under a range of possible atmospheric carbon dioxide levels. These include, 5 at the most extreme for 2030, "In summer and autumn, the range [of changes in rainfall] is typically $-15 \%$ to $+10 \%$ ". It is also stated that "In 2070 for the high emissions case, the range of annual rainfall change in central, eastern and northern areas [of Australia] grows to $-30 \%$ to $+20 \%$." and that seasonal rainfall decreases in some locations are predicted to be even more extreme.

10 There are various approaches to making projections of future runoff for changed climatic conditions, for example, precipitation and evapotranspiration. According to Sankarasubramanian et al. (2001), the most common approach is to use calibrated rainfall-runoff models with inputs changed from historical values to determine the resulting change in runoff. Typically this is achieved by scaling the historical values by 15 a constant factor. (We note that an alternative approach is to modify a rainfall series by setting some percentage $x$ of non-zero rain events to zero, sometimes known as thinning the rainfall series by $x$ percent. This was considered only briefly in this paper as the runoff results obtained for rainfall series thinned by $10 \%$ were comparable to those obtained by scaling rainfall by 0.9 .) When considering the effect on runoff of changes in rainfall, this type of study has tended to focus on models for annual or monthly runoff however this paper considers modeling runoff on a daily time step.

A reading of the literature suggests calibrating a rainfall-runoff model on a catchment and using it to predict future runoff for changed historical rainfall inputs may provide more information on the model than the catchment. For example, consider the re-

25 mark of Sankarasubramanian et al., "Climate sensitivity analyses performed on the same basin using different conceptual watershed models can lead to significantly different results." (Sankarasubramanian et al., 2001, page 1771). A broader comment made by Todini noted that apart from comparison of some conceptual rainfall-runoff models in the 1970s, "... no objective comparisons using benchmarks, or test beds

\section{HESSD}

8, 917-955, 2011

\section{Comparison of rainfall-runoff models in the Murray-Darling Basin}

J. M. Whyte et al.

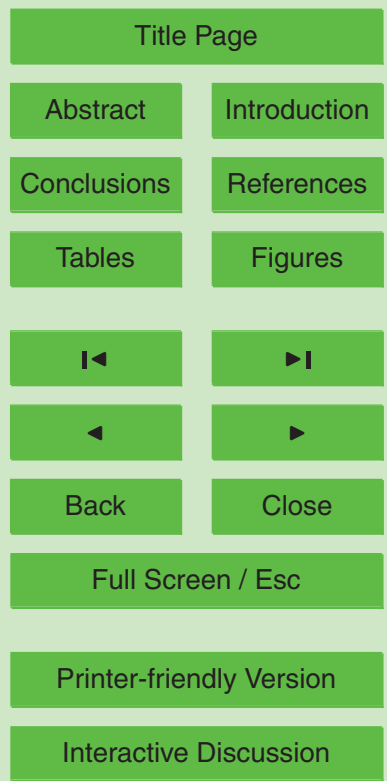


using standard data sets, have been proposed or effected." (Todini, 2007). This paper aims to contribute to a discussion on the comparison of rainfall-runoff models by using a selection of models with freely available data for Australia's Murray-Darling basin to make projections of runoff in selected catchments for rainfall changed from historical 5 values. Analysis of these results will illustrate the dependency of runoff projections on the model used and allow an assessment of whether catchments exhibit particular sensitivity to changes in rainfall regardless of the model used. Results are compared with those from a recently proposed empirical method for associating changes in rainfall with changes in runoff (Whyte, 2011).

10 Two forms of rainfall-runoff model are considered: the widely used lumped conceptual model SIMHYD and a selection of time series models which feature lagged runoff and rainfall terms. The version of SIMHYD used is that found in the eWater Rainfall Runoff Library (CRC for Catchment Hydrology, 2004) which permits free use of SIMHYD. This version of SIMHYD has nine parameters and predicts runoff given contemporaneous rainfall and evapotranspiration inputs. Some of the time series models considered are AutoRegressive with eXogenous input (ARX), also known as transfer function models (e.g., Beven, 2001) and Box-Jenkins models (Castellano-Méndez et al., 2004), and variants containing nonlinear autoregressive runoff terms (NARX) and a model for a logarithmic transform of runoff. A finite impulse response (FIR) model, that is, a time series model which features only lagged rainfall terms is also considered. These are all termed single regime time series models. A threshold autoregressive model with exogenous input (TARX) of two regimes provides a time series model capable of greater adaptation to the prevailing catchment conditions compared to the single regime time series models. The time series models are implemented in the open source programming language $\mathrm{R}$ and are applied to four MDB catchments. Models are calibrated and validated for each catchment using daily data, Nash-Sutcliffe coefficients are determined, and based on these results models which perform well are considered further.
HESSD

8, 917-955, 2011

\section{Comparison of rainfall-runoff models in the Murray-Darling Basin}

J. M. Whyte et al.

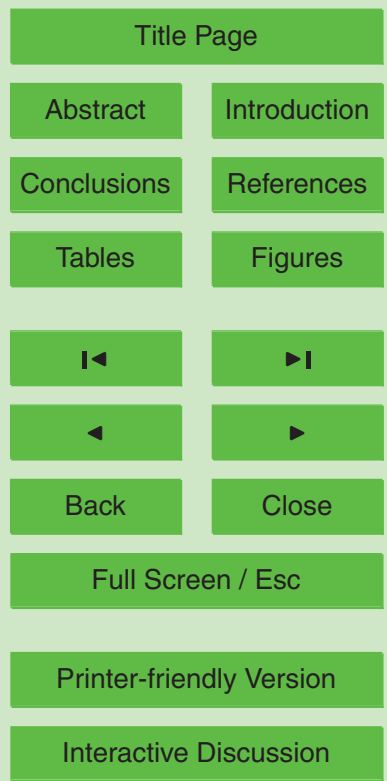


To consider the response of models to rainfall changed from historical levels, calibrated models are used to simulate runoff when the catchment rainfall data is scaled. Scaling factors that increase and decrease the rainfall are considered. SIMHYD in the Rainfall Runoff Library allows deterministic simulations, the programs written for the 5 time series models consider deterministic simulations, as performed for SIMHYD, and also stochastic simulations, that is, those which include a random noise term in the projections at each time step. Projected runoff scaling factors are determined using the model response for each rainfall scaling factor across the catchments, models and simulation methods considered. Where the nature of a time series model allows, a the10 oretical scaling of runoff for each rainfall scaling factor is determined from the model and parameter values of the fitted model.

This paper is organized as follows. Section 2 introduces the various time series models and SIMHYD, describes the MDB catchment data used and the calibration and validation of models and gives details of the simulation methods used to illustrate the behaviour of the rainfall-runoff models to scaled rainfall inputs. Some brief remarks on thinning of rainfall series are also given. The methodology of making comparisons of the responses is discussed in Sect. 3. Section 4 shows the calibration and validation results for each of the models for all four catchments and determines which of the time series models warrant further consideration. These models and SIMHYD are calibrated for each catchment using the appropriate entire data set. Section 5 uses the calibrated models to simulate runoff for each catchment when the historical catchment rainfall is increased and decreased by two, five, ten and twenty percent and quantifies the change in runoff according to the methodology of Sect. 3. Projections for SIMHYD and one of the selected time series models are considered for all four catchments using
HESSD

8, 917-955, 2011

Comparison of rainfall-runoff models in the Murray-Darling Basin

J. M. Whyte et al.

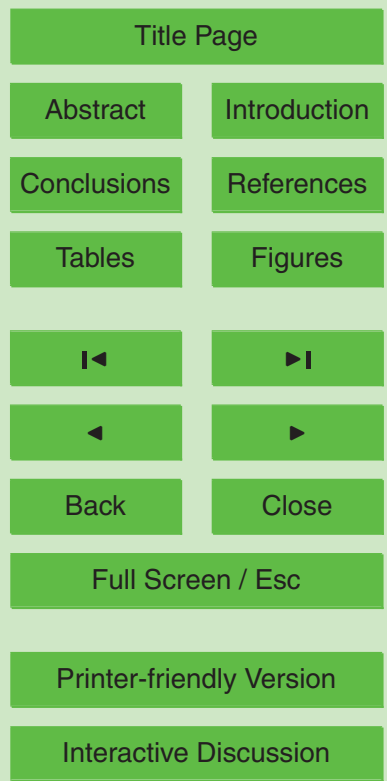




\section{Preliminaries}

We begin with an overview of SIMHYD as this model provides a reference point for comparison of the time series model results. Further, the input requirements of SIMHYD inform the choice of inputs for the other models. Models 1 to 6 represent

5 a variety of single regime time series models and the two regime threshold autoregressive model is defined subsequently.

\subsection{Models under consideration}

\subsubsection{SIMHYD}

SIMHYD, a simplified version of HYDROLOG (Porter and McMahon, 1971), is widely 10 used in modelling catchment runoff in Australia and elsewhere and is classified as a lumped conceptual model. This study uses the nine parameter version of SIMHYD implemented in the eWater Rainfall Runoff Library (CRC for Catchment Hydrology, 2004). The version of SIMHYD described by the Rainfall Runoff Library documentation (Podger, 2004) is capable of simulating runoff values for daily or monthly time steps.

15 When used on a daily time step, SIMHYD uses daily values of catchment potential evapotranspiration for each month and daily catchment rainfall values to calculate daily runoff. The model is calibrated against daily runoff data. The model features three connected storages, the first intercepts rainfall and the other two are each associated with a variable representing the amount of water in the storage and a parameter that defines the storage capacity. Catchment rainfall and water in the storages is distributed between storages and runoff according to deterministic rules which depend on the volumes in the storages, parameter values and inputs. The rules aim to model the dependence of water transport on the history of the climate variables in the catchment. As a result, the output of SIMHYD is strongly non-linear in the inputs. to a catchment on a daily time step are three daily catchment values; derived rainfall, estimated potential evapotranspiration and measured runoff.

HESSD

8, 917-955, 2011

Comparison of rainfall-runoff models in the Murray-Darling Basin

J. M. Whyte et al.

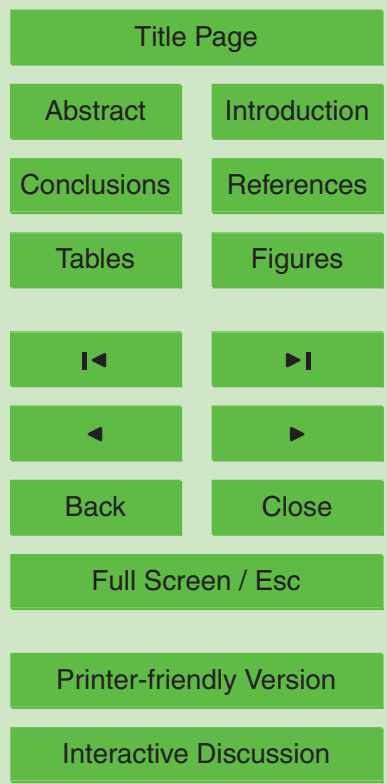




\subsubsection{ARX models}

In the following time series models $Y_{t-k}$ represents the runoff term of lag $k$ and $R_{t-1}$ the rainfall term of lag $I$, where $k$ and $/$ are non-negative integers. The time index $t$ starts from 1 . In all models $\varepsilon$ denotes a random variation of independent variates of mean 5 zero.

Model 1: (6 parameters: runoff lag $k=1$, rainfall lag $I=0,1$.) Fitting this model to data shows the quality of fit possible with a simple model. Also, this model provides a benchmark for comparisons. The cosine and sine terms define sinusoidal variation with a period of one year, which is a proxy for evapotranspiration and any other sea-

$Y_{t}=f_{1}(R, Y, t)+\varepsilon_{t}$

$f_{1}(R, Y, t)=\beta_{0}+\beta_{1} \cos \left(\frac{2 \pi t}{365.25}\right)+\beta_{2} \sin \left(\frac{2 \pi t}{365.25}\right)+\beta_{3} Y_{t-1}+\beta_{4} R_{t}+\beta_{5} R_{t-1}$

Models 2 and 3 allow an investigation of the effects of inclusion of additional predictor terms in Model 1 on the quality of fit.

Model 2: (8 parameters: runoff lag $k=1,2$, rainfall lag $I=0,1,2$.)

$$
Y_{t}=f_{2}(R, Y, t)+\varepsilon_{t},
$$

$f_{2}(R, Y, t)=f_{1}(R, Y, t)+\beta_{6} Y_{t-2}+\beta_{7} R_{t-2}$.

Model 3: (11 parameters: Model 2 plus squared lagged rainfall terms.)

$\begin{aligned} Y_{t} & =f_{3}(R, Y, t)+\varepsilon_{t} \\ f_{3}(R, Y, t) & =f_{2}(R, Y, t)+\beta_{8} R_{t}^{2}+\beta_{9} R_{t-1}^{2}+\beta_{10} R_{t-2}^{2}\end{aligned}$

\subsubsection{Models non-linear in runoff}

Model 4 is non-linear in the runoff terms and hence is a NARX model.

\section{HESSD}

8, 917-955, 2011

Comparison of rainfall-runoff models in the Murray-Darling

Basin

J. M. Whyte et al.

Title Page

Abstract

Introduction

Conclusions

References

Tables

Figures

14

4

Back

Close

Full Screen / Esc

Printer-friendly Version

Interactive Discussion 
Model 4: (14 parameters: Model 3 plus quadratic and cross product lagged runoff terms.)

$$
\begin{aligned}
Y_{t} & =f_{4}(R, Y, t)+\varepsilon_{t}, \\
f_{4}(R, Y, t) & =f_{3}(R, Y, t)+\beta_{11} Y_{t-1}^{2}+\beta_{12} Y_{t-2}^{2}+\beta_{13} Y_{t-1} Y_{t-2} .
\end{aligned}
$$

5 The dynamics of this model are non-linear and this can lead to instability which is apparent in simulations.

Transforming the runoff, by taking logarithm or some power for example (Beven, 2001, Page 250), gives a non-linear relationship between runoff and rainfall but the criterion for the stability of the corresponding autoregressive model is unchanged.

10 Model 5: (6 parameters: log transform of runoff, runoff lag $k=1$, rainfall $\operatorname{lag} l=0,1,2$.)

$$
\ln \left(Y_{t}+\exp (1)\right)=f_{5}(R, Y, t)+\varepsilon_{t}
$$

$$
\begin{aligned}
f_{5}(R, Y, t)= & \beta_{0}+\beta_{1} \cos \left(\frac{2 \pi t}{365.25}\right)+\beta_{2} \sin \left(\frac{2 \pi t}{365.25}\right)+\beta_{3} \ln \left(Y_{t-1}+\exp (1)\right) \\
& +\beta_{4} R_{t}+\beta_{5} R_{t-1}
\end{aligned}
$$

When calculating fitted values or making predictions, the value of runoff is determined 15 from (1) by

$$
Y_{t}:=\max \left(\exp \left[\ln \left(Y_{t}+\exp (1)\right)\right]-\exp (1), 0\right)
$$

which limits predictions of runoff to non-negative values. It is necessary to add a positive value to the runoff before taking the logarithm because there are zero values in the recorded time series for various catchments. After some experimentation, the value $\exp (1)$ was found to give fitted values that were much closer to observations than those determined for smaller positive values.

HESSD

8, 917-955, 2011

Comparison of rainfall-runoff models in the Murray-Darling Basin

J. M. Whyte et al.

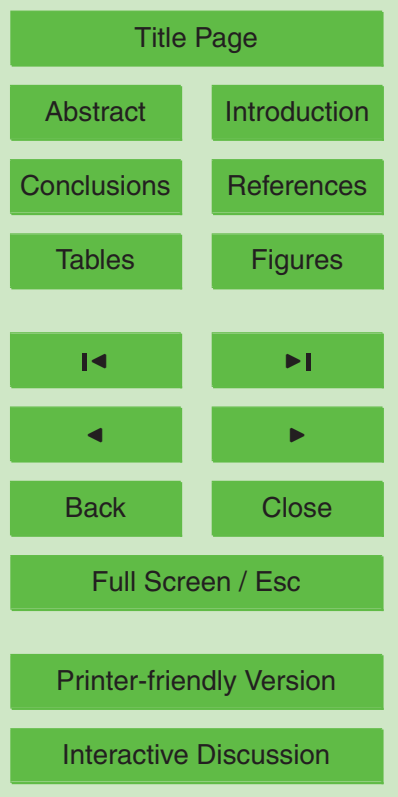




\subsubsection{The Finite Impulse Response (FIR) model}

This type of model does not depend on previous runoff.

HESSD

Model 6: $(N+3$ parameters, rainfall lags $I=0, \ldots, N)$

8, 917-955, 2011

$$
Y_{t}=f_{6}(R, Y, t)+\varepsilon_{t},
$$

${ }_{5} f_{6}(R, Y, t)=\sum_{l=0}^{N} \beta_{l} R_{t-l}+\beta_{N+2} \cos \left(\frac{2 \pi t}{365.25}\right)+\beta_{N+3} \sin \left(\frac{2 \pi t}{365.25}\right)$.

In preliminary studies it was determined that increasing $N$ beyond 40 gave no improvement to the fit of the model to data for the catchments considered. Also if an intercept term was included its estimate was negative for all catchments. Since this is inconsistent with a physical interpretation of an intercept as a baseflow, it was excluded from 10 the model.

\subsubsection{A two regime threshold autoregressive model}

The models used here to predict runoff are similar to the open-loop threshold autoregressive systems given in Tong (1995, page 101) and are termed TARX models to emphasize that the rainfall is an exogenous input. In preliminary studies it was found that an autoregressive threshold model for runoff tended to produce runs of negative fitted values. To prevent such counter physical results it was decided to trial a TARX model for log transformed runoff with a single threshold. The model is given by

$\ln \left(Y_{t}+c\right)= \begin{cases}f^{[1]}(R, Y, t)+\varepsilon_{t}, & R_{t}<\tau \\ f^{[2]}(R, Y, t)+\varepsilon_{t}, & R_{t} \geq \tau\end{cases}$

where for $i=1,2$,

${ }_{20} f^{[i]}(R, Y, t)=\beta_{0}^{[i]}+\beta_{1}^{[i]} \ln \left(Y_{t-1}+c\right)+\beta_{2}^{[i]} R_{t}$,

where $c$ plays the same role as $\exp (1)$ in the log runoff model (1).

Comparison of rainfall-runoff models in the Murray-Darling Basin

J. M. Whyte et al.

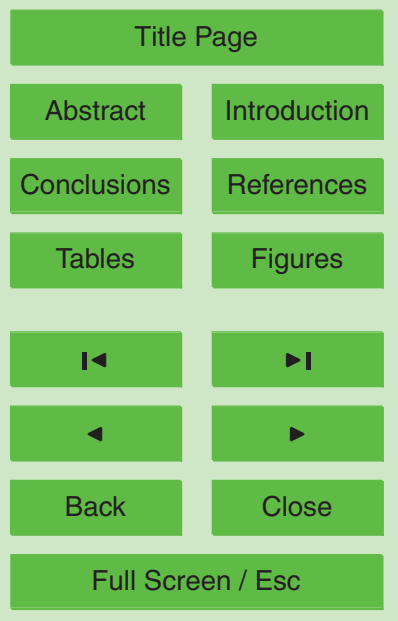

Printer-friendly Version

Interactive Discussion 
When calculating fitted values or making predictions, the value of runoff is determined from (2) by

$Y_{t}:=\exp \left[\ln \left(Y_{t}+c\right)\right]-c$.

Seasonal variation is not explicitly included, and we rely on rainfall to make some im5 plicit allowance.

\subsection{Calibration and validation of the models}

\subsubsection{Overview}

Using a data set that contains missing data values poses a complication for the use of time series models. It is necessary to either fit models in a piecemeal fashion or infill missing data before fitting a model. This issue is avoided if it is possible to find a subset of a data set that does not contain missing values which is long enough to be useful. At this point it is convenient to introduce a definition for such a subset.

Definition 2.1. A working data set for a catchment is the data belonging to a continuous period of time for which both daily rainfall and daily runoff are available. By definition, this set cannot have any missing values.

Model calibration is a process which adjusts the values of the model parameters to ensure that the fitted runoff values produced by the model are close to the observed runoff values according to some specified criterion. In this paper a model is calibrated by determining the parameter values that minimize the residual sum of squares (RSS) where for data $y_{i}$ and fitted values $\hat{y}_{i}, i=1, \ldots, n$ the RSS is $\sum_{i=1}^{n}\left(y_{i}-\hat{y}_{i}\right)^{2}$.

Model validation follows calibration. Validating a model requires the use of the model with parameter values determined by calibration to predict runoff for that portion of the data excluded from the model calibration stage. For any day, the calibrated model provides a prediction that is one step ahead in terms of runoff, but uses rainfall up to
HESSD

8, 917-955, 2011

Comparison of rainfall-runoff models in the Murray-Darling Basin

J. M. Whyte et al.

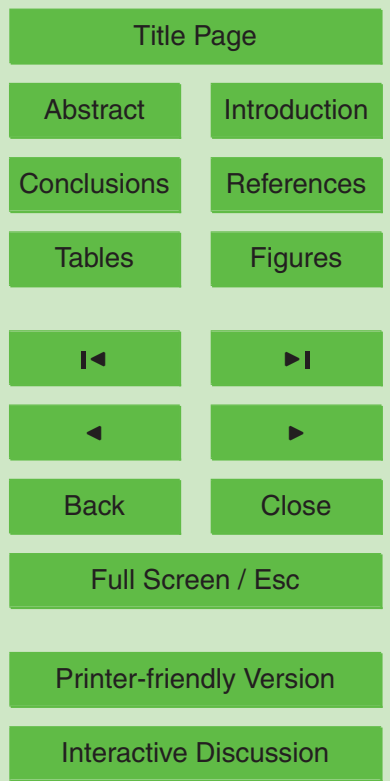


Each model considered on a particular catchment (except for the TARX models) is calibrated on approximately the first two thirds of the catchment working data and validated for runoff on the remaining one third.

For both the model calibration and validation it is useful to determine the degree 5 of agreement between the data and fitted values as poor agreement suggests that the model does not capture the essential features of the system and is unsuitable for making predictions. The agreement of data and fitted values is quantified by calculating a Nash-Sutcliffe model efficiency coefficient (Nash and Sutcliffe, 1970) for each of the model calibration and validation stages. For mean observed runoff $\bar{y}$, the Nash-

$$
E=1-\frac{\sum_{i=1}^{n}\left(y_{i}-\hat{y}_{i}\right)^{2}}{\sum_{i=1}^{n}\left(y_{i}-\bar{y}\right)^{2}},
$$

which is the coefficient of determination $\left(R^{2}\right)$ in the statistics literature. Larger values of $E$ show a better agreement between fitted and observed values. The maximum value of $E$ is one, which occurs when each fitted runoff value is equal to the corresponding observed value. In this paper $E_{c}$ is used to denote the Nash-Sutcliffe coefficient for model calibration and $E_{\mathrm{v}}$ that for validation. The symbol $E_{\mathrm{r}}$ is used for the NashSutcliffe coefficient determined from a calibration of a model over the entire appropriate working data set, termed here a recalibration.

\subsubsection{Calibration and validation of single regime time series models}

Calibration and recalibration of models was performed by the use of the $\operatorname{Im}()$ function in R. Model validation followed the scheme outlined in the preliminary comments.

\subsubsection{Calibration of TARX models}

Calibration of the TARX model used on each catchment was with the routine TVAR() (Stigler, 2010) available in the tsDyn package in $\mathrm{R}$ which fits a multivariate threshold 927
HESSD

8, 917-955, 2011

Comparison of rainfall-runoff models in the Murray-Darling Basin

J. M. Whyte et al.

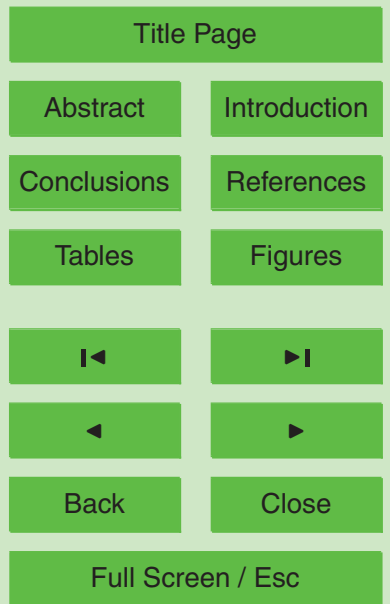

Printer-friendly Version

Interactive Discussion 
model to data. This approach was chosen as the function provided for fitting a univariate model does not allow for an exogenous variable, rainfall in this case. So, a bivariate model for runoff and rainfall was fitted. However, only the runoff model is required for the comparisons made here. Given these complications in fitting, the TARX models 5 were fitted to the entire record only.

\subsubsection{Calibration and validation of SIMHYD}

SIMHYD determines warm-up periods for both calibration and validation. The response of SIMHYD is nonlinear in the parameters. Model calibration proceeds by an iterative process for minimizing the residual sum of squares (RSS). In the Rainfall Runoff Library the optimizer used to minimize the RSS was initially pattern search multi-start with ten starts, followed by application of the Rosenbrock single start optimizer until the RSS did not appear to change in at least the third decimal place.

\subsection{Data}

This paper draws on daily values of catchment rainfall, daily catchment runoff and 15 monthly potential evapotranspiration collated for a National Land and Water Resources Audit (Peel et al., 2000). This CSIRO data was obtained from the "child records" linked to the Audit report (Peel et al., 2000) accessed from the Australian Natural Resources Database (http://adl.brs.gov.au/anrdl/php/anrdlSearch.html). The catchment daily rainfall was supplied by the Queensland Department of Natural Resources and Mining and the process followed is described in (Jeffrey et al., 2001). Briefly, values supplied are determined by interpolation of rainfall data between measurement stations to give rainfall values on a grid of 0.05 degree cells and then averaging these cell values over cells in the catchment of interest. The catchment runoff data used to calibrate SIMHYD is that ascribed to an Australian Bureau of Meteorology (ABoM) flow station in the catch-
HESSD

8, 917-955, 2011

Comparison of rainfall-runoff models in the Murray-Darling Basin

J. M. Whyte et al.

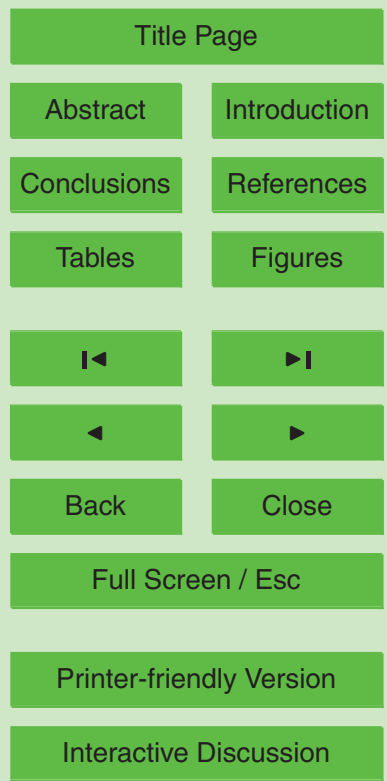


Some features of catchments used in this study and the data used are given in Table 1. The flow station names for catchments one to four are: (1) Fifteen mile creek at Greta south, (2) Jingellic creek at Jingellic, (3) Ovens river at Bright, and (4) Mannus creek at Tooma.

$5 \quad$ The calibration data used for all four catchments contained the highest runoff period and Catchment 2's calibration data also contained the lowest runoff period, as determined by the Rainfall Runoff Library software.

\section{Methodology for exploring the response of rainfall-runoff models to scaled rainfall}

10 Published studies have classified the suitability of a calibrated model by making the general comment that a Nash-Sutcliffe coefficient $E$ greater than 0.6 "suggest a reasonable modelling of runoff" and an $E$ value greater than 0.8 "suggest a good modelling of runoff for catchment yield studies" (see, for example, Chiew and Siriwardena, 2005). In this study, following calibration and validation all models are calibrated over their 15 entire working data set for each catchment for completeness and the resultant NashSutcliffe coefficient is denoted by $E_{\mathrm{r}}$. Models which show Nash-Sutcliffe coefficients generally greater than 0.6 are considered under scaled rainfall conditions.

Following this discrimination step, a recalibrated model of interest is used for multi step ahead prediction of runoff for each catchment with modified historical rainfall. Two methods are used to achieve this.

1. Historical rainfall is scaled by factors that increase and decrease the input rainfall by two, five, ten and twenty percent. That is, factors $0.8,0.9,0.95,0.98(1.02$, $1.05,1.1$ and 1.2) are used to reduce (increase) the volume of rainfall. This approach is applied to all models that are deemed as worth further consideration.
Models are applied to all four catchments.
HESSD

8, 917-955, 2011

Comparison of rainfall-runoff models in the Murray-Darling Basin

J. M. Whyte et al.

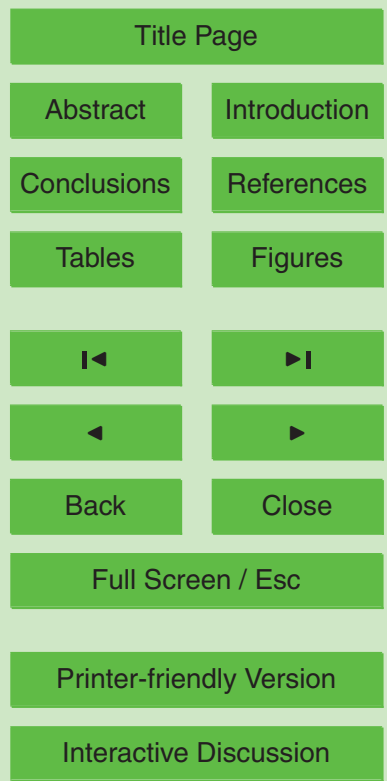


2. For the purposes of comparison, for all four catchments the rainfall series is thinned and one time series model and SIMHYD are considered. This thinning is achieved by using a series of pseudo-random numbers to set approximately $10 \%$ of the non-zero rain values in the historical record to zero.

\section{5}

The runoff predictions calculated using modified historical rainfall are multi step ahead in the lagged streamflows but use modified rainfall for the day of, and typically days preceding, the prediction.

\subsection{Deterministic predictions}

The version of SIMHYD implemented in the Rainfall Runoff Library behaves as a deterministic model. In SIMHYD applying a scaling factor to an input historical rainfall series is achieved by using the data scaling feature and setting the rainfall multiplier for each month to the desired value. The time series models from Sect. 2.1 under consideration can be used as deterministic models by setting the noise terms $(\varepsilon)$ to their expected value of zero.

15 Remark 1. Autoregressive runoff models of lag $L \geq 1$ in runoff require $L$ runoff data values to predict a runoff value at the $(L+1)$ th time point, however runoff data corresponding to the scaled rainfall is not available. The approach taken here is to use historical runoff values for the lagged runoff terms required to initiate the prediction of runoff. The effect of the initial values is transient for stable models. To ensure that the runoff predictions are not unduly affected by the choice of initial runoff values, we check that after the first 100 time steps the effect of the initial values is transient and therefore has negligible effect on the predictions for all of the models considered. This is achieved by scaling initial values by the first calculated runoff scaling factor, recalculating the runoff scaling factor and comparing this second value with the first runoff scaling value.

Suppose the recalibration of a model on catchment $j$ gives coefficients of lagged runoff terms which correspond to a stable model. For catchment $j$ the runoff series 930
HESSD

8, 917-955, 2011

Comparison of rainfall-runoff models in the Murray-Darling Basin

J. M. Whyte et al.

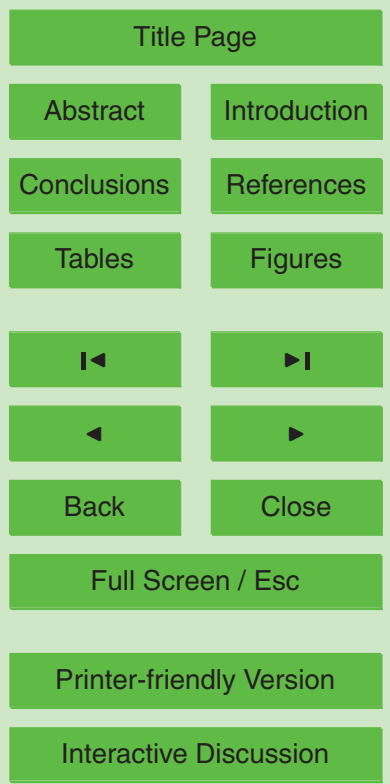


simulated by the model for historical rainfall scaled by a factor $\alpha>0$ is denoted by $\left\{\hat{y}_{i}^{[j, \alpha]}\right\}_{i=1}^{n}$. The runoff scaling factor resulting from a rainfall scaling factor $\alpha$ for this model on catchment $j$ is defined by

$\hat{S}(j, \alpha)=\frac{\sum_{i=100}^{n} \hat{y}_{i}^{[j, \alpha]}}{\sum_{i=100}^{n} \hat{y}_{i}^{[j, 1]}}$

5 and, by Remark 1, is practically independent of the initial runoff values. The denominator of (4) shows that the response of the model to scaled input rainfall is compared to the response predicted by using the historical rainfall, that is, $\alpha=1$.

Calculation of a predicted runoff scaling factor resulting from an increase in the number of zero rain days in the thinned series compared to the historical rainfall follows

10 a similar convention. Suppose $\beta<1$ represents the proportion of non-zero rain days in the historical series that are retained in the thinned series. For a given model on catchment $j$, the runoff series simulated by the model for historical rainfall thinned by $\beta$ percent is denoted by $\left\{\dot{y}_{i}^{[j, \beta]}\right\}_{i=1}^{n}$. The runoff scaling factor resulting from a rainfall thinning factor $\beta$ for this model on catchment $j$ is defined by

${ }_{15} \grave{S}(j, \beta)=\frac{\sum_{i=100}^{n} \grave{y}_{i}^{[j, \beta]}}{\sum_{i=100}^{n} \hat{y}_{i}^{[j, 1]}}$.

\subsection{Stochastic predictions}

If a model meets the conditions required for its use in deterministic predictions then it is possible to proceed to stochastic predictions. When using a single regime time series model of Sect. 2.1 as a stochastic model it is necessary to determine an approby inspecting the residuals returned by recalibration of the model on the appropriate catchment.

HESSD

8, 917-955, 2011

Comparison of rainfall-runoff models in the Murray-Darling Basin

J. M. Whyte et al.

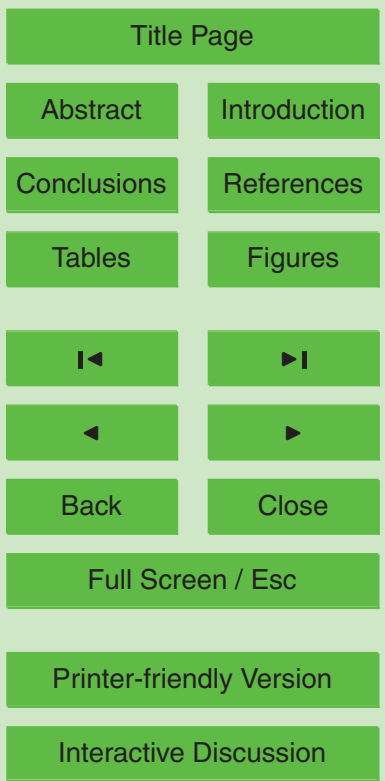


In a similar vein to the discussion of deterministic predictions, for catchment $j$ the runoff series arising from a model's stochastic simulation of runoff with historical rainfall scaled by a factor $\alpha>0$ is denoted by $\left\{\tilde{y}_{i}^{[j, \alpha]}\right\}_{i=1}^{n}$ and the corresponding runoff scaling factor is

${ }_{5} \tilde{S}(j, \alpha)=\frac{\sum_{i=100}^{N} \tilde{y}_{i}^{[j, \alpha]}}{\sum_{i=100}^{N} \tilde{y}_{i}^{[j, 1]}}$.

\subsection{Theoretical runoff scaling values}

Given an ARX model of interest and historical rainfall for a catchment, taking the expected value of both sides of the relation and rearranging gives an expression for mean runoff $\bar{Y}$ in terms of mean rainfall $\bar{R}$ and model parameters. Replacing $\bar{R}$ by $\alpha \bar{R}$ gives the model's prediction for $\bar{Y}_{\alpha}$, mean runoff for mean historical rainfall scaled by $\alpha$. The quotient $\bar{Y}_{\alpha} / \bar{Y}$ is the model's theoretical runoff scaling for a rainfall scaling $\alpha$ on this catchment.

To give an illustration of this process, consider Model 1 for catchment $j$. Rearrangement of the model expression gives

${ }_{15} Y_{t}-\beta_{3} Y_{t-1}=\beta_{0}+\beta_{1} \cos \left(\frac{2 \pi t}{365.25}\right)+\beta_{2} \sin \left(\frac{2 \pi t}{365.25}\right)+\beta_{4} R_{t}+\beta_{5} R_{t-1}$.

Replacing runoff and rainfall terms by their historical means $\bar{Y}$ and $\bar{R}$, respectively, noting that the expected value of the sine and cosine terms is zero and rearranging gives

$\bar{Y}=\frac{\beta_{0}+\left(\beta_{4}+\beta_{5}\right) \bar{R}}{\left(1-\beta_{3}\right)}$ and catchment $j$ is
HESSD

$8,917-955,2011$

Comparison of rainfall-runoff models in the Murray-Darling

Basin

J. M. Whyte et al.

\section{Title Page}

Abstract

Introduction

Conclusions

References

Tables

Figures
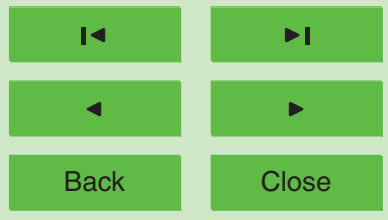

Full Screen / Esc

Printer-friendly Version

Interactive Discussion 
$S(j, \alpha)=\frac{\beta_{0}+\left(\beta_{4}+\beta_{5}\right) \alpha \bar{R}}{\beta_{0}+\left(\beta_{4}+\beta_{5}\right) \bar{R}}$.

HESSD

We note that for $\beta_{0}$ in (7),

8, 917-955, 2011

if $\left\{\begin{array}{c}\beta_{0} \gtrless 0, \alpha \lessgtr 0, \\ \beta_{0}=0, \\ \beta_{0} \lessgtr 0, \alpha \lessgtr 0,\end{array}\right.$ then $S(j, \alpha)\left\{\begin{array}{l}< \\ =\alpha . \\ >\end{array}\right.$

Expressions for theoretical rainfall scalings for Models 2 and 3 follow similarly. Note 5 that the calculation for Model 3 requires the expected value of the squared rainfall, $E\left[R^{2}\right]=\bar{R}^{2}+\sigma_{R}^{2}$, where $\sigma_{R}^{2}$ represents the variance of the catchment rainfall data. In the case of Model 5 it is not as straightforward to obtain a theoretical rainfall scaling as for the other models. As Model 6 is a FIR model and hence is independent of past runoff values and linear in rainfall with no intercept term, scaling rainfall by a factor $\alpha$ results

10 in a scaling of runoff by $\alpha$. Given the predictable nature of this behaviour, Model 6 is not considered further.

\section{Model calibration and validation results}

\subsection{Results for SIMHYD}

The results of the calibration and validation of SIMHYD are presented in Table 2. SIMHYD produces very similar $E_{\mathrm{c}}$ and $E_{\mathrm{v}}$ pairs for all four catchments. These results show that the runoff predictions of SIMHYD in the validation phase are respectable. The validation of SIMHYD suggests that it is reasonable to calibrate SIMHYD for each catchment over the catchment's entire working data set for subsequent consideration.

Remark 2. For all SIMHYD calibrations the value of the pervious fraction parameter is very close to one, which means the proportion of rainfall directed to runoff along the impervious path is very small. As a result it seems that the nine parameter model

Comparison of rainfall-runoff models in the Murray-Darling Basin

J. M. Whyte et al.

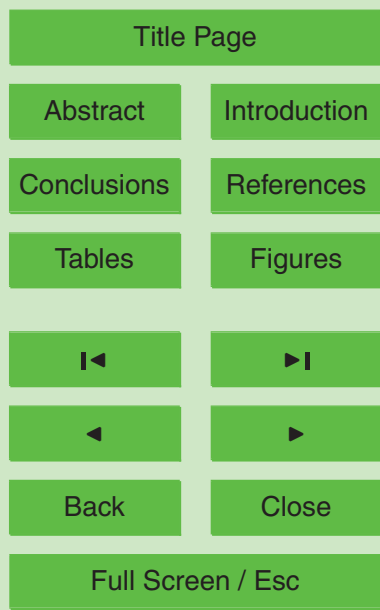

Printer-friendly Version

Interactive Discussion 
must have very similar behaviour to the seven parameter version of SIMHYD for these catchments.

\subsection{Calibration and validation of the single regime time series models}

The Nash-Sutcliffe coefficients of the model calibrations and validations are presented 5 in Table 3. The Nash-Sutcliffe coefficient must increase if we add more parameters to a model and estimate all of the parameters from the data. However, given the long time series, even increases in the third decimal place are statistically significant.

Table 3 shows that Models 1 and 2 have very similar Nash-Sutcliffe values across the four catchments considered. Model 3 performs marginally better than Models 1 and 2 in three catchments and noticeably better in Catchment 1 . Model 4 produces better Nash-Sutcliffe values than Model 3, with slightly higher $E_{\mathrm{c}}$ and $E_{\mathrm{r}}$ values for all catchments and substantially better $E_{\mathrm{v}}$ values for Catchments 1,2 and 4 . The Model 5 $E_{\mathrm{c}}$ and $E_{\mathrm{r}}$ values are comparable to those seen for Models 3 and 4 . Model 5's $E_{\mathrm{v}}$ values are smaller than those seen for Model 4 but still acceptable. The FIR model, Model 6, is substantially poorer than the other models at modelling runoff and is not considered further.

Given the similarity of results for Models 1 and 2, only Model 1 is considered further in the determination of model response to scaled input rainfall. Model 3 is considered as it has five more parameters than Model 1 and has the potential to exhibit different behaviour in the runoff simulations. The results of Models 4 and 5 justify further consideration to gain some insight into the properties of models using squares and products of lagged runoff and log transform of runoff terms, respectively.

\subsection{Calibration of TARX models}

The Nash-Sutcliffe coefficients for the calibrations are given in Table 4.

Stand-out features of Tables 3 and 4 are that across all time series models considered, the $E_{\mathrm{r}}$ value is the lowest for Catchment 4 and highest for Catchment 3.
HESSD

8, 917-955, 2011

Comparison of rainfall-runoff models in the Murray-Darling Basin

J. M. Whyte et al.

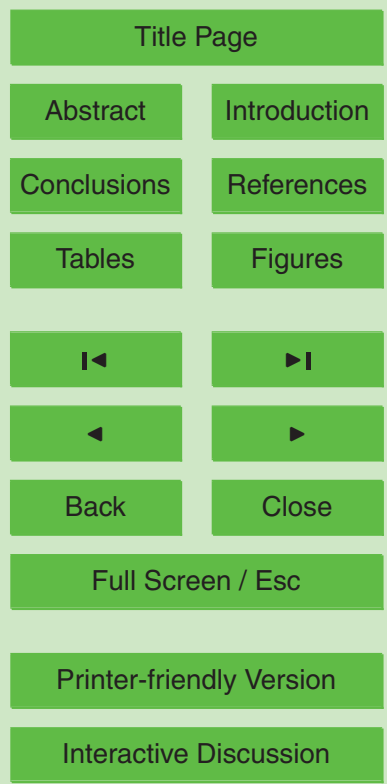




\section{Model response to scaled rainfall input}

It is known that NARX models are generally unstable, (see, for example, Fan and Yao, 2005, pages 125-126)) and the use of Model 4 for multi-step ahead prediction of runoff for one catchment showed that this was the case here. As a result, Model 4 is not 5 considered further. In all cases runoff scalings are rounded to three decimal places.

\subsection{Simulation of runoff by deterministic models}

In the following discussion $\Downarrow$ shows that runoff scaling factor is closer to one than the rainfall scaling factor and hence the effect is attenuated. Conversely, $\Uparrow$ shows that runoff scaling factor is further from one than the rainfall scaling factor and hence the effect is amplified.

\subsubsection{SIMHYD projections}

Results are presented in Table 5.

From Table 5 some general features are evident:

For $\alpha>1, \quad \alpha<\hat{S}(3, \alpha)<\hat{S}(2, \alpha) \lesssim \hat{S}(1, \alpha)<\hat{S}(4, \alpha)$.

For $\alpha<1, \quad \hat{S}(4, \alpha)<\hat{S}(2, \alpha) \lesssim \hat{S}(1, \alpha)<\hat{S}(3, \alpha)<\alpha$.

15 The results of Eq. (8) show that in all cases the effect of the rainfall scaling $\alpha$ is amplified in the runoff scaling. The response of the runoff to a scaling in rainfall is greatest for Catchment 4 (greatest deviation of the $\hat{S}$ value from 1) and least for Catchment 3 (smallest deviation of the $\hat{S}$ value from 1 ) regardless of the $\alpha$ value used.

When considering the $10 \%$ thinned rainfall series, $\grave{S}(1,0.9)=0.792, \grave{S}(2,0.9)=0.717$, $\grave{S}(3,0.9)=0.763$ and $\grave{S}(4,0.9)=0.776$. This gives the ordering

$$
\underbrace{\grave{S}(2,0.9)<\grave{S}(3,0.9)<\grave{S}(4,0.9)<\grave{S}(1,0.9)}_{\Downarrow},
$$

HESSD

8, 917-955, 2011

Comparison of rainfall-runoff models in the Murray-Darling Basin

J. M. Whyte et al.

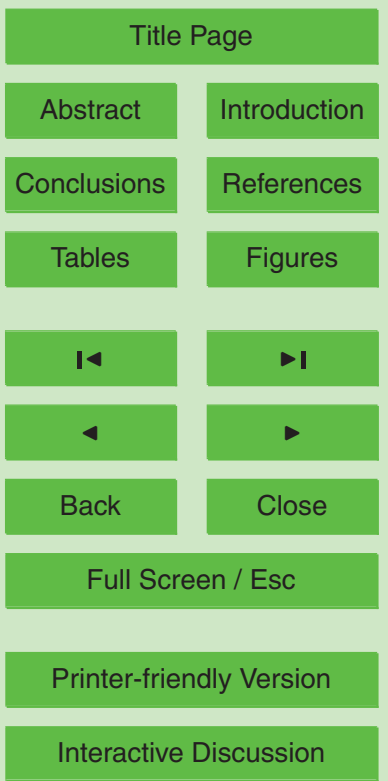


which is different to the ordering observed for $\alpha=0.9$ in Eq. (8) but all $S(j, 0.9)$ in Eq. (9) are within 0.04 of their corresponding $\hat{S}(j, 0.9)$ value in Table 5 . Given this similarity the effects of the thinned rainfall series are not considered further in this paper.

\subsubsection{Single regime time series model projections}

5 Following the discussion of Sect. 3, the behaviour of selected time series models is assessed for scaled rainfall inputs, checking in each case that the stability assumption of Remark 1 is satisfied. Results for Models 1, 3 and 5 following Eq. (4) are shown in Tables 7, 8 and 9, respectively.

For Models 1, 3 and 5 it is possible to make some general comments on the runoff scalings which follow from the deterministic simulations.

Model 1 results are given in Eq. (10).

For $\alpha \gtrless 1, \quad \underbrace{\hat{S}(2, \alpha) \lessgtr \hat{S}(1, \alpha)}_{\Downarrow} \lessgtr \alpha \lessgtr \underbrace{\hat{S}(4, \alpha) \lessgtr \hat{S}(3, \alpha)}_{\Uparrow}$.

A particular case of the results for Model 1 in Eq. (10) is examined in detail to illustrate the results obtained. For $\alpha=0.8$, Table 7 shows that $\hat{S}(3,0.8)=0.750$ is less than $\hat{S}(4,0.8)=0.792$ and both values are less than the rainfall scaling factor, indicating an amplification of the effect of the rainfall scaling on the runoff. Further, $\hat{S}(1,0.8)=0.844$ is less than $\hat{S}(2,0.8)=0.863$ and both values are greater than 0.8 showing that the rainfall scaling has an attenuated effect on runoff.

An overall statement that follows from Eq. (10) is that Catchment 3's runoff is most influenced by rainfall scaling, Catchment 2's runoff is the least influenced. The ordering of the deterministic runoff scalings in Eq. (10) is the same as that observed for the theoretical runoff scaling values in Table 7.

The runoff scaling factors resulting from the $10 \%$ thinned rainfall series for Model 1 are $\grave{S}(1,0.9)=0.927, \grave{S}(2,0.9)=0.919, \grave{S}(3,0.9)=0.870$ and $\grave{S}(4,0.9)=0.898$. This gives
HESSD

8, 917-955, 2011

Comparison of rainfall-runoff models in the Murray-Darling Basin

J. M. Whyte et al.

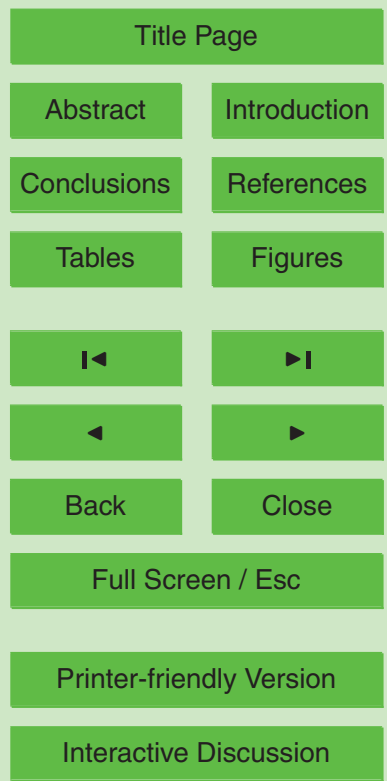


$\underbrace{\grave{S}(3,0.9)<\grave{S}(4,0.9)}_{\Uparrow}<\underbrace{\grave{S}(2,0.9)<\grave{S}(1,0.9)}_{\Downarrow}$,

which is not the same as seen in Eq. (10) as Catchments 1 and 2 have swapped positions, but does show the same attenuation and amplification of the rainfall scaling as seen in Eq. (10). Moreover, each $\grave{S}(j, 0.9)$ is within 0.013 of its corresponding $\hat{S}(j, 0.9)$ 5 value in Table 7.

Model 3 results are given in Eq. (12).

$$
\begin{array}{ll}
\alpha>1: & \underbrace{\hat{S}(2, \alpha)<\hat{S}(4, \alpha)}_{\Downarrow}<\alpha<\underbrace{\hat{S}(1, \alpha)<\hat{S}(3, \alpha)}_{\Uparrow}, \\
\alpha<1: & \underbrace{\hat{S}(3, \alpha)}_{\Uparrow}<\alpha<\underbrace{\hat{S}(4, \alpha) \lesssim \hat{S}(1, \alpha)<\hat{S}(2, \alpha)}_{\Downarrow} .
\end{array}
$$

Compared to the ordering of the theoretical runoff scalings seen in Table 8, for most values of $\alpha$ the ordering of the deterministic scalings given in Eq. (12) is the same. In all 10 cases Catchment 3 is most influenced by rainfall scaling, Catchment 2 least influenced. The results in Eq. (12) show that the behaviour of the catchments is not symmetric with respect to increases and decreases in rainfall scaling $\alpha$.

Model 5 results are given in Eq. (13).

$\alpha \gtrless 1: \underbrace{\hat{S}(2, \alpha) \lessgtr \hat{S}(4, \alpha) \approx \hat{S}(1, \alpha)}_{\Downarrow} \lessgtr \alpha \lessgtr \underbrace{\hat{S}(3, \alpha)}_{\Uparrow}$.

15 The results of Eq. (13) show again that Catchment 3 is most affected by a rainfall scaling and Catchment 2 least affected.

\subsubsection{TARX results}

Through the process of obtaining runoff scaling factors for the calibrated TARX models it became clear that the results depend significantly on the value of $c$ used. It was

HESSD

8, 917-955, 2011

Comparison of rainfall-runoff models in the Murray-Darling Basin

J. M. Whyte et al.

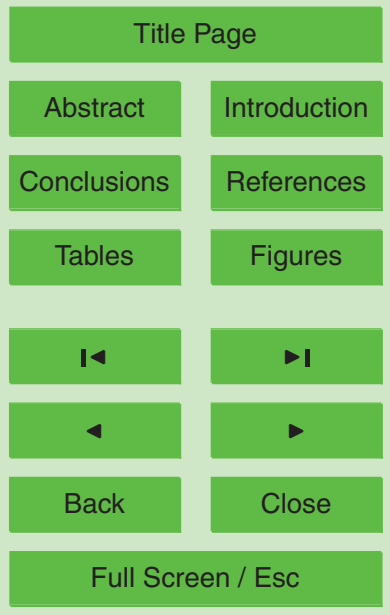

Printer-friendly Version

Interactive Discussion 
noted that increasing $c$ in the calibration of Eq. (2) tended to increase the number of negative fitted values as well as $E_{\mathrm{r}}$ and could produce runoff scaling values which were not sensible in some cases. Through some preliminary experimentation it was decided to accept lower Nash-Sutcliffe coefficients for the calibrations if the $c$ value 5 used eliminated the occurrence of negative fitted values or at least produced a relatively small number of negative values that were quite close to zero.

Runoff scalings are presented in Table 6.

Trends from Table 6 are shown in (14).

$\left.\begin{array}{l}\text { For } \alpha<1, \hat{S}(3, \alpha) \lesssim \hat{S}(1, \alpha)<\hat{S}(2, \alpha)<\hat{S}(4, \alpha)<\alpha \\ \text { For } \alpha>1, \alpha<\hat{S}(4, \alpha)<\hat{S}(2, \alpha)<\hat{S}(1, \alpha)<\hat{S}(3, \alpha)\end{array}\right\} \Uparrow$

10 Note that for all values of $\alpha$ and all catchments the effect of the rain scaling is amplified in the runoff scaling. For $\alpha>1$ the order of runoff scalings from greatest distance from one to least distance is Catchment 3, then 1, then 2 then 4 . For $\alpha<1$ the ordering is reversed. This shows that using the TARX model, Catchment 3 is the most sensitive to runoff scaling and Catchment 4 the least sensitive.

\subsubsection{Overview of results}

The features that stand out for the TARX and the single regime time series models is that Catchment 3 presents as the catchment which has the greatest amplification of the rainfall scaling factor in the effect on runoff. SIMHYD results show that Catchment 4 shows the greatest effect from rainfall scaling with Catchment 3 showing the least effect. This shows that there is not complete agreement between the models on the catchments that are most and least sensitive to rainfall scaling. SIMHYD and the TARX models have the feature that the rainfall scaling is always amplified in the runoff scaling.

To obtain an appreciation for how the runoff scalings change with the model used, 25 a graph of the range of runoff scalings obtained from deterministic predictions is given in Fig. 1. This figure shows that SIMHYD amplifies the effect of the runoff scaling the 938
HESSD

8, 917-955, 2011

Comparison of rainfall-runoff models in the Murray-Darling Basin

J. M. Whyte et al.

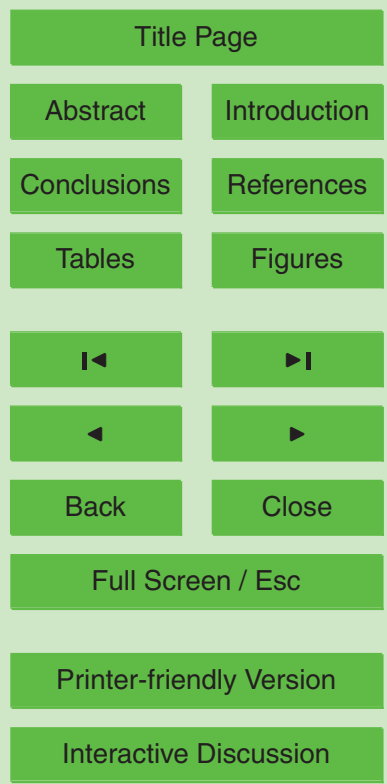


most followed by the TARX model. There is not a great difference between the results obtained for the single regime time series models. The results become more greatly spread as the rainfall scaling takes values further from one.

\subsection{Simulation of runoff by stochastic models}

5 Each stochastic runoff simulation required selection of an appropriate distribution for the random noise added in the time series models. In each case a plot of residuals from a model recalibration was used to decide on appropriate features for the noise distribution for the model. For Models 1 and 3, residual plots showed that the spread of the residuals appeared to increase as the fitted value increases and that the distribution of residuals is positively skewed. These features suggest the use of Gumbel extreme value type I distributions (henceforth referred to as Gumbel distributions for brevity) where the scale parameter used depends on the fitted value. Details of the simulation of random variates from these distributions are given in Appendix $A$.

For Model 5, considering the positive and absolute values of the negative residuals 15 separately suggested that in each case random noise was appropriately modelled by an exponential distribution. In preparation for the stochastic simulations for a specific catchment the parameters of the exponential distribution were determined for the positive and negative residuals. The proportion of negative residuals to the total number of residuals, $p_{\mathrm{n}}$, was determined. The generation of random noise for each time step for 20 the catchment of interest proceeded by first drawing a random variate from the unit uniform distribution. If this value was less than $p_{\mathrm{n}}$ the random noise was generated from the distribution for the negative residuals and subtracted from the prediction of the deterministic model. Otherwise, the random variate was drawn from the distribution for the positive residuals and added to the deterministic model prediction.
HESSD

8, 917-955, 2011

Comparison of rainfall-runoff models in the Murray-Darling Basin

J. M. Whyte et al.

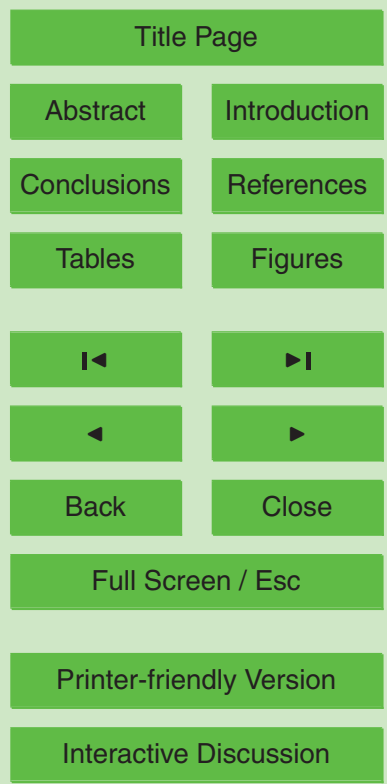




\subsubsection{Discussion of results}

For Model 1 the runoff scalings as defined by Eq. (6) obtained from the stochastic simulations did not change the ordering of the results seen for the theoretical or deterministic results.

5 Results for Model 3 are quite similar to those seen for the deterministic runoff scalings and are given in Eq. (15).

For $\alpha>1, \quad \underbrace{\tilde{S}(2, \alpha)<\tilde{S}(4, \alpha)}_{\Downarrow}<\alpha \lesssim \underbrace{\tilde{S}(3, \alpha) \lesssim \tilde{S}(1, \alpha)}_{\Uparrow}$

For $\alpha<1, \quad \alpha \lesssim \underbrace{\tilde{S}(3, \alpha)<\tilde{S}(1, \alpha) \lesssim \tilde{S}(4, \alpha)<\tilde{S}(2, \alpha)}_{\Downarrow}$.

Equation (15) shows that for $\alpha>1$ the results for Catchments 1 and 3 are now very similar. Also, for $\alpha<1$ the effect of rainfall scaling is attenuated for each catchment.

10 This is due to the increase of the runoff scaling determined for Catchment 3 by a value which is quite large in the context of the results obtained. In absolute terms, Catchment 3 shows the greatest difference between the runoff scalings determined from deterministic and stochastic methods, however this difference is only 0.021 at its largest.

For Model 5 the ordering of the runoff scalings obtained from the stochastic simulations is essentially the same as seen in Eq. (13) with a greater difference between the results of Catchments 4 and 1 . Catchment 4 shows the greatest difference between the runoff scalings obtained for the deterministic and stochastic runoff simulations, but this difference at its greatest is only 0.026 .

\section{Conclusions}

Different rainfall-runoff models may produce very similar degrees of agreement between observed and predicted runoff values as seen (for example) by comparison of Nash-Sutcliffe coefficients. However, the models can give quite different projections of
HESSD

8, 917-955, 2011

Comparison of rainfall-runoff models in the Murray-Darling Basin

J. M. Whyte et al.

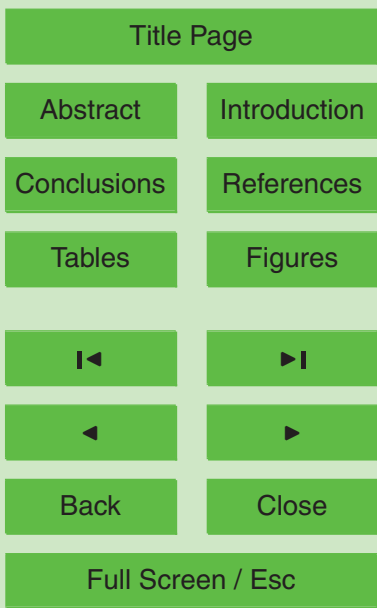

Printer-friendly Version

Interactive Discussion 
runoff when the inputs change. In particular the conceptual model SIMHYD typically amplifies changes in mean rainfall by a factor of between two and three whereas the single regime regression type models typically show slight amplification or attenuation depending on the catchment. The amplification of the rainfall change observed for 5 SIMHYD is consistent with Young's claims that a $1 \%$ change in rainfall leads to a $2-3 \%$ change in runoff (see, for example, Young and McColl, 2009) and Whyte's empirical study (Whyte, 2011). The regression type models are useful for short-term predictions as might be used in day to day control algorithms.

An explanation for the unrealistic performance of the regression type models is that 10 they represent a Taylor series approximation around the mean value of runoff in the data set to which they are fitted with either just linear terms or with linear terms predominating. A change in rainfall has the consequence that the Taylor series is used for predictions further from the mean value used in the fitting process and the linear approximation deteriorates. There is also the issue that increased rainfall may (rain 15 scaling values of $\alpha>1$ ) may involve some linear extrapolation of the regression models. In summary, the approximate linear scaling of runoff relative to rainfall input is an artifact of the model and should not be taken to represent the physical process. The two regime regression models (TARX) circumvent this limitation to some extent and using more regimes would provide further flexibility however the nonlinear nature of the models makes calibration less straightforward, particularly if nonnegative predictions are required. We note that the predictions from the TARX models are much closer to those of SIMHYD than the single regime regression models.

The differences between runoff scaling factors obtained from theoretical, deterministic and stochastic methods were negligible. This is expected for the linear models but if there are nonlinearities the variance and higher moments play a part. It seems that these effects tend to cancel in the method used here for quantifying the effect on runoff of a rainfall change. Given the variability of rainfall inputs, the stochastic simulation seems the most realistic, so the finding suggests that precise modelling of the distribution of residuals may not be necessary.

\section{HESSD}

8, 917-955, 2011

\section{Comparison of rainfall-runoff models in the Murray-Darling Basin}

J. M. Whyte et al.

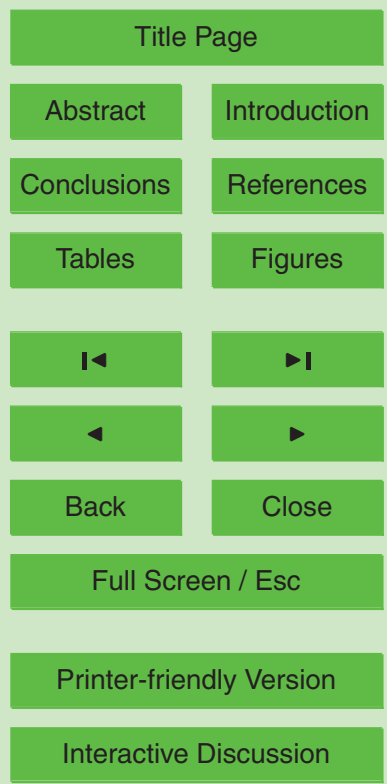


Our limited investigation suggests that the consequences of a $10 \%$ thinning of rainfall are very similar to a $10 \%$ reduction in rainfall introduced by a scaling factor.

Our results suggest that if models are to be used as part of climate change scenario studies it is essential that the effect of rainfall change is investigated in addition to $R^{2}$.

\section{Appendix A}

\section{Simulation of pseudo-random variates from the Gumbel extreme value type I distribution}

We will use $\tilde{y}_{i}$ as the simulated runoff from a stochastic prediction model as

$10 \tilde{y}_{i}=\hat{y}_{i}+\varepsilon_{i}, \quad \varepsilon_{i} \sim \operatorname{Gumbel}\left(\xi_{i}, \theta_{i}\right), \quad E\left[\varepsilon_{i}\right]=0$.

The use of Eq. (A1) requires estimates of $\xi_{i}$ and $\theta_{i}$ which we denote as $\hat{\xi}_{i}$ and $\hat{\theta}_{i}$.

The Gumbel cumulative distribution function (c.d.f) with parameters $\xi$ and $\theta$ given by

$F(x)=\exp \left(-\exp \left[-\left(\frac{x-\xi}{\theta}\right)\right]\right)$,

has mean

$15 \mu=\xi+\gamma \theta$

(where the Euler-Mascheroni constant $\gamma \approx 0.5772$ ) and standard deviation

$\sigma=\theta \frac{\pi}{\sqrt{6}}$.

To generate a random variate from a Gumbel distribution as given by Eq. (A2), for random variate $u \sim U[0,1]$ (where $U[0,1]$ is the uniform distribution on the interval $[0,1]$ ) we assume $F(x)=u$ in Eq. (A2) and rearrange to give

$x=\xi+\theta(-\ln (-\ln u)), \quad x \sim \operatorname{Gumbel}(\xi, \theta)$.
HESSD

8, 917-955, 2011

Comparison of rainfall-runoff models in the Murray-Darling

Basin

J. M. Whyte et al.

Title Page

Abstract

Introduction

Conclusions

References

Tables

Figures

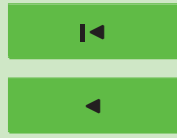

$\rightarrow$

Back

Close

Full Screen / Esc

Printer-friendly Version

Interactive Discussion 
When random variates are required for use as additive noise in a regression model it is assumed that $\mu=0$.

For a Gumbel distribution of mean $\mu=0$, rearrangement of Eq. (A3) gives $\xi=-\gamma \theta$,

5 and hence we may adapt Eq. (A5) to give the random variable $X \sim \operatorname{Gumbel}(-\gamma \theta, \theta)$ $X=\theta(-\gamma+(-\ln (-\ln U))), U \sim U[0,1], \quad E[X]=0$.

From relation $(\mathrm{A} 7)$ note that $E[|X|]=\theta E[|(-\gamma+(-\ln (-\ln U)))|]$.

Consider the variable $X_{1} \sim \operatorname{Gumbel}(-\gamma, 1)$. Using $\theta=1$ in (A8) and estimating $E\left[\left|X_{1}\right|\right]$ by 10 simulation to obtain $\bar{x}_{\theta=1}$ gives

$E[|(-\gamma+(-\ln (-\ln U)))|] \approx \bar{x}_{\theta=1}$

and hence where $X \sim \operatorname{Gumbel}(-\gamma \theta, \theta)$ for unknown $\theta$, this parameter is estimated by

$\hat{\theta}=\frac{E[|X|]}{\bar{x}_{\theta=1}}$.

In this study $\bar{x}_{\theta=1}$ was determined from simulation as 0.984 .

Our variables of interest are $\varepsilon_{i}$, assumed distributed as Gumbel $\left(-\gamma \theta_{i}, \theta_{i}\right)$. Adapting Eq. (A9) we have

$\hat{\theta}_{i}=\frac{E\left[\left|\varepsilon_{i}\right|\right]}{\bar{x}_{\theta=1}}$.

In this study we choose to model $E\left[\left|\varepsilon_{i}\right|\right]$ by proposing a linear regression of residuals $\left|r_{i}\right|$ against $\hat{y}_{i}$ :

$20 \quad\left|r_{i}\right|=\hat{\alpha}_{0}+\hat{\alpha}_{1} \hat{y}_{i}+\epsilon_{i}, \quad E\left[\epsilon_{i}\right]=0$

and hence $E\left[\left|\varepsilon_{i}\right|\right]=\hat{\alpha}_{0}+\hat{\alpha}_{1} \hat{y}_{i}$.

Given $E\left[\left|\varepsilon_{i}\right|\right], \hat{\theta}_{j}$ is found by Eq. (A10). Using $\hat{\theta}_{i}$ in Eq. (A7) gives a random variate from Gumbel $\left(\hat{\xi}_{i}, \hat{\theta}_{i}\right)$ for use in Eq. (A1). Continue for all $i$.

HESSD

8, 917-955, 2011

Comparison of rainfall-runoff models in the Murray-Darling Basin

J. M. Whyte et al.

Title Page

Abstract Introduction

Conclusions

References

Tables

Figures

14

4

Back

Close

Full Screen / Esc

Printer-friendly Version

Interactive Discussion 
Acknowledgements. The authors acknowledge support from the Australian Research Council, Discovery Project grant DP0877707.

HESSD

8, 917-955, 2011

\section{References}

Beven, K. J.: Rainfall-Runoff Modelling: The Primer, John Wiley \& Sons, Ltd., Chichester, England, 2001. 920, 924

Castellano-Méndez, M., González-Manteiga, W., Febrero-Bande, M., Manuel PradaSánchez, J., and Lozano-Calderón, R.: Modelling of the monthly and daily behaviour of the runoff of the Xallas river using Box-Jenkins and neural networks methods, J. Hydrol., 296, 38-58, 2004. 920

Chiew, F. H. S. and Siriwardena, L.: Estimation of SIMHYD Parameter Values For Application In Ungauged Catchments, in: MODSIM 2005, 2883-2889, 2005. 929

CRC for Catchment Hydrology: The Rainfall Runoff Library, release 1.0.5, www.toolkit.net.au/ Tools/RRL (last access: December 2010), 2004. 920, 922

CSIRO and ABS: Climate change in Australia, Tech. Rep., CSIRO and the Australian Bureau of Meteorology, www.climatechangeinaustralia.gov.au (last access: November 2009), 2007. 919

CSIRO and the Australian Bureau of Meteorology: Climate change in Australia. Observed changes and projections, projections developed by CSIRO and the Australian Bureau of Meteorology for the Australian Climate Change Science Programme, 2007. 919

Fan, J. and Yao, Q.: Nonlinear Time Series: Nonparametric and Parametric Methods, Springer, New York, USA, 2005. 935

Jeffrey, S. J., Carter, J. O., Moodie, K. B., and Beswick, A. R.: Using spatial interpolation to construct a comprehensive archive of Australian climate data, Environ. Modell. Softw., 16, 309-330, doi:10.1016/S1364-8152(01)00008-1, http://www.sciencedirect.com/science/ article/B6VHC-42SXF8F-2/2/b5c0a24b85149f40ba5d2419ddc0999c (last access: December 2010), 2001. 928

Nash, J. E. and Sutcliffe, J. V.: River flow forecasting through conceptual models part I - a discussion of principles, J. Hydrol., 10, 282-290, 1970. 927

Peel, M. C., Chiew, F. H. S., Western, A. W., and McMahon, T. A.: National Land and Water Resources Audit Theme 1 - Water Availability Extension of Unimpaired Monthly Streamflow

\section{Comparison of rainfall-runoff models in the Murray-Darling Basin}

J. M. Whyte et al.

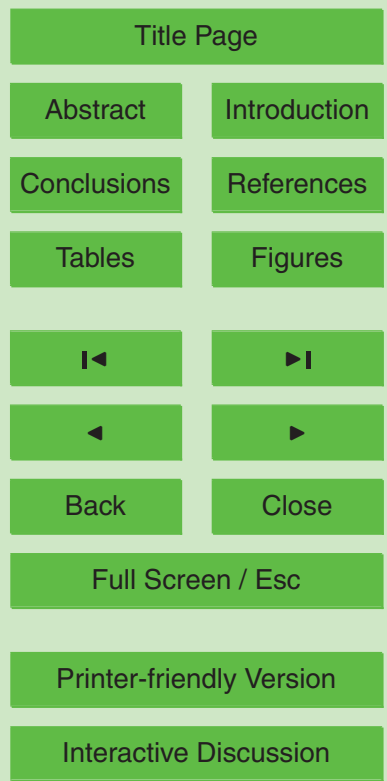


Data and Regionalisation of Parameter Values to Estimate Streamflow in Ungauged Catchments, Tech. Rep., National Land and Water Resources Audit, 2000. 928

Podger, G.: Rainfall Runoff Library Users Guide, Department of Infrastructure, Planning and Natural Resources; CRC for Catchment Hydrology, revision 1.0.5, issued 21 June 2004, 2004. 922

Porter, J. W. and McMahon, T. A.: A model for the simulation of streamflow data from climatic records, J. Hydrol., 13, 297-324, 1971. 922

Sankarasubramanian, A., Vogel, R. M., and Limbrunner, J. F.: Climate elasticity of streamflow in the United States, Water Resour. Res., 37, 1771-1781, 2001. 919

10 Stigler, M.: TVAR, multivariate threshold model fitting in $R$, version 0.7-40, 2010. 927

Todini, E.: Hydrological catchment modelling: past, present and future, Hydrol. Earth Syst. Sci., 11, 468-482, doi:10.5194/hess-11-468-2007, 2007. 920

Tong, H.: Non-linear Time Series: A Dynamical System Approach, Oxford University Press, New York, 1995.

Whyte, J. M.: A discussion of variability of results from an empirical process for relating changes in runoff to changes in rainfall, submitted for publication in the proceedings of the 34th International Association for Hydro-Environment Engineering and Research World Congress, Brisbane 2011, in review, 2011. 920, 941

Young, M. and McColl, J.: Theres a hole in the bucket Dear Liza, Dear Liza, a hole!, third Tuesday Lecture, Singapore Campus, The University of Adelaide, 19 May 2009, Slides accessed from http://www.myoung.net.au/water/talks.php, last access: January 2010. 941

\section{HESSD}

8, 917-955, 2011

\section{Comparison of rainfall-runoff models in the Murray-Darling Basin}

J. M. Whyte et al.

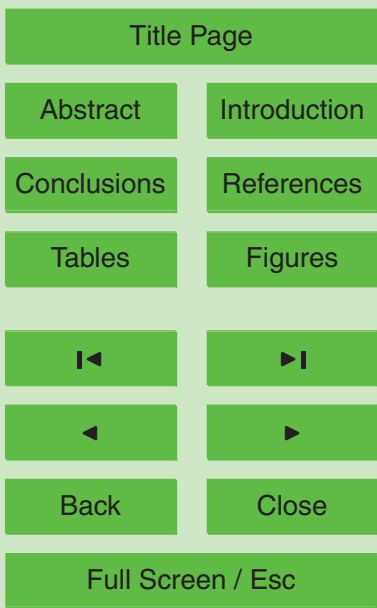

Printer-friendly Version

Interactive Discussion 


\section{HESSD}

8, 917-955, 2011

\section{Comparison of rainfall-runoff models in the Murray-Darling Basin}

Table 1. For each of the four catchments studied the table shows the area, Australian Bureau of Meteorology (ABoM) flow station present and the number of days used in the calibration $\left(n_{\mathrm{c}}\right)$ and validation $\left(n_{\mathrm{v}}\right)$ of models.

\begin{tabular}{ccccc}
\hline \multicolumn{2}{c}{ Catchment } & Flow station & & \\
$\#$ & area $\left(\mathrm{km}^{2}\right)$ & ABoM number & $n_{\mathrm{c}}$ & $n_{\mathrm{v}}$ \\
\hline 1 & 229 & 403213 & 8036 & 3712 \\
2 & 378 & 401013 & 6309 & 3241 \\
3 & 495 & 403205 & 10957 & 5784 \\
4 & 504 & 401008 & 5484 & 3524 \\
\hline
\end{tabular}

Title Page

\section{Abstract}

Conclusions

\section{Tables}

14

4

Back

Full Screen / Esc

Printer-friendly Version

Interactive Discussion 


\section{HESSD}

8, 917-955, 2011

\section{Comparison of rainfall-runoff models in the Murray-Darling Basin}

Table 2. Nash-Sutcliffe coefficient of determination for calibration and validation of SIMHYD, $\Delta E=E_{\mathrm{c}}-E_{\mathrm{v}}$ and the Nash-Sutcliffe coefficient for the recalibration of SIMHYD $\left(E_{\mathrm{r}}\right)$ to three decimal places for the four catchments studied.

\begin{tabular}{ccccc}
\hline Catchment \# & $E_{\mathrm{c}}$ & $E_{\mathrm{v}}$ & $\Delta E$ & $E_{\mathrm{r}}$ \\
\hline 1 & 0.790 & 0.812 & -0.022 & 0.807 \\
2 & 0.758 & 0.679 & 0.079 & 0.701 \\
3 & 0.784 & 0.774 & 0.010 & 0.786 \\
4 & 0.736 & 0.745 & -0.009 & 0.749
\end{tabular}

Title Page

\section{Abstract}

Conclusions

Tables

14

4

Back

Full Screen / Esc

Printer-friendly Version 
Table 3. Nash-Sutcliffe coefficient of determination for calibration and validation of ARX models (Models 1-3), NARX Model 4, log transform of runoff Model 5 and an FIR model (Model 6) for the four catchments studied.

\begin{tabular}{cccccc}
\hline \multicolumn{2}{c}{ Catchment } & 1 & 2 & 3 & 4 \\
\hline \multirow{3}{*}{ Model 1 } & $E_{\mathrm{c}}$ & 0.736 & 0.651 & 0.941 & 0.584 \\
& $E_{\mathrm{v}}$ & 0.806 & 0.665 & 0.957 & 0.616 \\
& $E_{\mathrm{r}}$ & 0.775 & 0.657 & 0.946 & 0.608 \\
\hline \multirow{3}{*}{ Model 2 } & $E_{\mathrm{c}}$ & 0.740 & 0.652 & 0.943 & 0.585 \\
& $E_{\mathrm{v}}$ & 0.817 & 0.667 & 0.961 & 0.618 \\
& $E_{\mathrm{r}}$ & 0.779 & 0.659 & 0.949 & 0.609 \\
\hline \multirow{4}{*}{ Model 3 } & $E_{\mathrm{c}}$ & 0.814 & 0.670 & 0.948 & 0.590 \\
& $E_{\mathrm{v}}$ & 0.802 & 0.662 & 0.964 & 0.623 \\
& $E_{\mathrm{r}}$ & 0.822 & 0.670 & 0.953 & 0.614 \\
\hline \multirow{3}{*}{ Model 4 } & $E_{\mathrm{c}}$ & 0.830 & 0.692 & 0.950 & 0.611 \\
& $E_{\mathrm{v}}$ & 0.544 & 0.424 & 0.852 & 0.381 \\
& $E_{\mathrm{r}}$ & 0.835 & 0.689 & 0.955 & 0.634 \\
\hline \multirow{3}{*}{ Model 5 } & $E_{\mathrm{c}}$ & 0.796 & 0.690 & 0.950 & 0.643 \\
& $E_{\mathrm{v}}$ & 0.866 & 0.696 & 0.967 & 0.655 \\
& $E_{\mathrm{r}}$ & 0.827 & 0.698 & 0.955 & 0.664 \\
\hline \multirow{3}{*}{ Model 6 } & $E_{\mathrm{c}}$ & 0.509 & 0.458 & 0.621 & 0.373 \\
$(N=40)$ & $E_{\mathrm{v}}$ & 0.340 & 0.428 & 0.549 & 0.312 \\
& $E_{\mathrm{r}}$ & 0.549 & 0.449 & 0.627 & 0.398 \\
\hline
\end{tabular}




\section{HESSD}

8, 917-955, 2011

\section{Comparison of} rainfall-runoff models in the Murray-Darling

Basin

Table 4. Nash-Sutcliffe coefficients for the calibration of a TARX model (Eq. 2) using the entire catchment working data set for the four catchments considered.

\begin{tabular}{ccc}
\hline Catchment & $E_{\mathrm{r}}$ & $c$ in Eq. (2) \\
\hline 1 & 0.855 & 0.01 \\
2 & 0.665 & 0.01 \\
3 & 0.954 & 0.01 \\
4 & 0.551 & 0.8 \\
\hline
\end{tabular}

Title Page

Abstract

Conclusions

Tables

14

4

Back

Full Screen / Esc

Printer-friendly Version

Interactive Discussion 


\section{HESSD}

8, 917-955, 2011

Comparison of rainfall-runoff models in the Murray-Darling Basin

Table 5. Runoff scaling factors using SIMHYD for deterministic simulations for the four catchments considered with a range of values for rainfall scaling factor $\alpha$. All values are rounded to three decimal places.

\begin{tabular}{c|cccccccc}
\hline SIMHYD & \multicolumn{8}{c}{ Rainfall scaling $\alpha$} \\
Runoff scaling & 0.8 & 0.9 & 0.95 & 0.98 & 1.02 & 1.05 & 1.1 & 1.2 \\
\hline$\hat{S}(1, \alpha)$ & 0.554 & 0.756 & 0.873 & 0.948 & 1.053 & 1.134 & 1.275 & 1.579 \\
$\hat{S}(2, \alpha)$ & 0.545 & 0.754 & 0.873 & 0.949 & 1.052 & 1.133 & 1.272 & 1.577 \\
$\hat{S}(3, \alpha)$ & 0.557 & 0.764 & 0.879 & 0.951 & 1.050 & 1.126 & 1.256 & 1.519 \\
$\hat{S}(4, \alpha)$ & 0.527 & 0.741 & 0.864 & 0.944 & 1.058 & 1.149 & 1.314 & 1.712 \\
\hline
\end{tabular}

Title Page

Abstract

Introduction

J. M. Whyte et al.

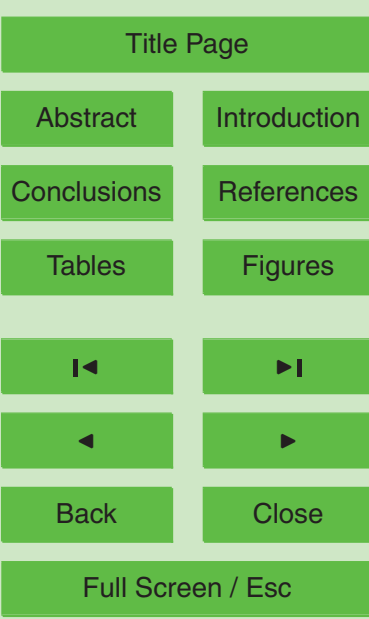

Printer-friendly Version

Interactive Discussion 


\section{HESSD}

8, 917-955, 2011

Comparison of rainfall-runoff models in the Murray-Darling Basin

Table 6. Runoff scaling factors using TARX model for deterministic simulations for the four catchments considered with a range of values for rainfall scaling factor $\alpha$. All values are rounded to three decimal places.

\begin{tabular}{c|cccccccc}
\hline TARX & \multicolumn{8}{c}{ Rainfall scaling $\alpha$} \\
runoff scaling & 0.8 & 0.9 & 0.95 & 0.98 & 1.02 & 1.05 & 1.1 & 1.2 \\
\hline$\hat{S}(1, \alpha)$ & 0.636 & 0.817 & 0.908 & 0.964 & 1.033 & 1.091 & 1.191 & 1.392 \\
$\hat{S}(2, \alpha)$ & 0.721 & 0.859 & 0.927 & 0.971 & 1.031 & 1.072 & 1.140 & 1.291 \\
$\hat{S}(3, \alpha)$ & 0.633 & 0.807 & 0.904 & 0.965 & 1.042 & 1.106 & 1.221 & 1.473 \\
$\hat{S}(4, \alpha)$ & 0.797 & 0.892 & 0.942 & 0.976 & 1.024 & 1.054 & 1.121 & 1.276 \\
\hline
\end{tabular}

Title Page

Abstract

Introduction

J. M. Whyte et al.

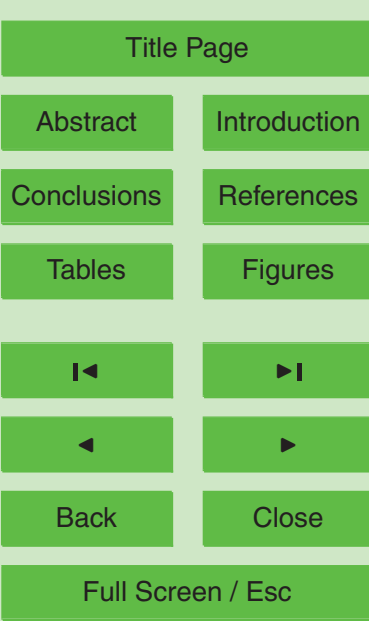

Printer-friendly Version

Interactive Discussion 


\section{HESSD}

8, 917-955, 2011

Table 7. Runoff scalings determined from theoretical considerations, $S($, ), deterministic $\hat{S}($, ) and stochastic $\tilde{S}($, ) simulations using Model 1 for the four catchments considered with a range of values for rainfall scaling factor $\alpha$. All values are rounded to three decimal points. A symbol $\uparrow_{k}\left(\downarrow_{k}\right)$ shows that the runoff scaling value is larger than (smaller than) the corresponding theoretical scaling factor by $k \times 0.001$. The $\sim$ show that a runoff scaling value is the same as its corresponding theoretical value to three decimal places.

\begin{tabular}{c|cccccccc}
\hline Model 1 & \multicolumn{8}{c}{ Rainfall scaling $\alpha$} \\
runoff scaling & 0.8 & 0.9 & 0.95 & 0.98 & 1.02 & 1.05 & 1.1 & 1.2 \\
\hline$S(1, \alpha)$ & 0.844 & 0.922 & 0.961 & 0.984 & 1.016 & 1.039 & 1.078 & 1.156 \\
$\hat{S}(1, \alpha)$ & $\uparrow_{1}$ & $\uparrow_{1}$ & $\sim$ & $\uparrow_{1}$ & $\downarrow_{1}$ & $\sim$ & $\downarrow_{1}$ & $\downarrow_{1}$ \\
$\tilde{S}(1, \alpha)$ & $\downarrow_{8}$ & $\downarrow_{4}$ & $\downarrow_{2}$ & $\sim$ & $\sim$ & $\uparrow_{2}$ & $\uparrow_{4}$ & $\uparrow_{8}$ \\
$S(2, \alpha)$ & 0.863 & 0.932 & 0.966 & 0.986 & 1.014 & 1.034 & 1.068 & 1.137 \\
$\hat{S}(2, \alpha)$ & $\uparrow_{1}$ & $\sim$ & $\sim$ & $\sim$ & $\sim$ & $\sim$ & $\sim$ & $\downarrow_{1}$ \\
$\tilde{S}(2, \alpha)$ & $\uparrow_{1}$ & $\sim$ & $\sim$ & $\sim$ & $\sim$ & $\sim$ & $\sim$ & $\downarrow_{1}$ \\
$S(3, \alpha)$ & 0.750 & 0.875 & 0.937 & 0.975 & 1.025 & 1.063 & 1.125 & 1.250 \\
$\hat{S}(3, \alpha)$ & $\sim$ & $\sim$ & $\uparrow_{1}$ & $\sim$ & $\sim$ & $\downarrow_{1}$ & $\sim$ & $\sim$ \\
$\tilde{S}(3, \alpha)$ & $\downarrow_{2}$ & $\downarrow_{1}$ & $\sim$ & $\sim$ & $\sim$ & $\sim$ & $\uparrow_{1}$ & $\uparrow_{2}$ \\
$S(4, \alpha)$ & 0.792 & 0.896 & 0.948 & 0.979 & 1.021 & 1.052 & 1.104 & 1.208 \\
$\hat{S}(4, \alpha)$ & $\downarrow_{1}$ & $\sim$ & $\sim$ & $\sim$ & $\sim$ & $\sim$ & $\uparrow_{1}$ & $\uparrow_{1}$ \\
$\tilde{S}(4, \alpha)$ & $\uparrow_{8}$ & $\uparrow_{4}$ & $\uparrow_{2}$ & $\uparrow_{1}$ & $\downarrow_{1}$ & $\downarrow_{2}$ & $\downarrow_{4}$ & $\downarrow_{8}$ \\
\hline
\end{tabular}

Comparison of rainfall-runoff models in the Murray-Darling Basin

J. M. Whyte et al.

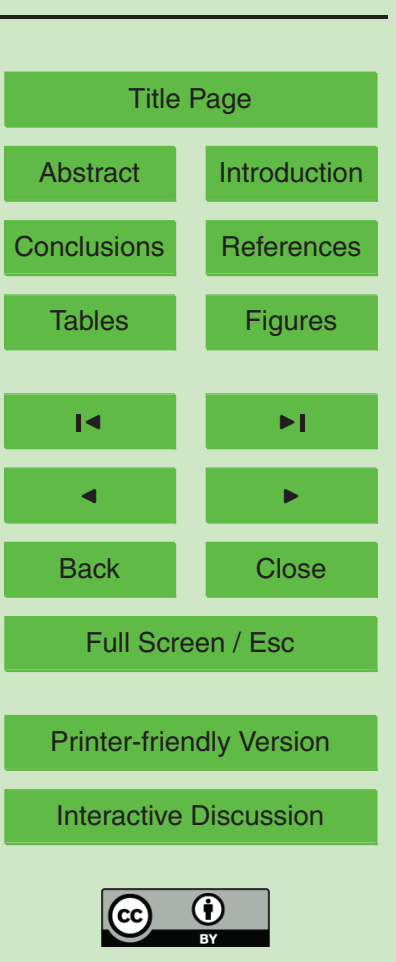




\section{HESSD}

8, 917-955, 2011

Table 8. Runoff scalings determined from theoretical relationships, deterministic and stochastic simulations using Model 3 for the four catchments considered with a range of values for rainfall scaling factor $\alpha$. All values are rounded to three decimal points. Symbols used are as for Table 7.

\begin{tabular}{c|cccccccc}
\hline Model 3 & \multicolumn{8}{|c}{ Rainfall scaling $\alpha$} \\
runoff scaling & 0.8 & 0.9 & 0.95 & 0.98 & 1.02 & 1.05 & 1.1 & 1.2 \\
\hline$S(1, \alpha)$ & 0.816 & 0.905 & 0.952 & 0.980 & 1.020 & 1.050 & 1.102 & 1.211 \\
$\hat{S}(1, \alpha)$ & $\uparrow_{1}$ & $\sim$ & $\sim$ & $\uparrow_{1}$ & $\sim$ & $\sim$ & $\sim$ & $\downarrow_{1}$ \\
$\tilde{S}(1, \alpha)$ & $\sim$ & $\downarrow_{1}$ & $\downarrow_{1}$ & $\sim$ & $\sim$ & $\sim$ & $\uparrow_{1}$ & $\uparrow_{1}$ \\
$S(2, \alpha)$ & 0.856 & 0.928 & 0.964 & 0.986 & 1.014 & 1.036 & 1.072 & 1.145 \\
$\hat{S}(2, \alpha)$ & $\uparrow_{1}$ & $\sim$ & $\sim$ & $\sim$ & $\sim$ & $\sim$ & $\sim$ & $\downarrow_{1}$ \\
$\tilde{S}(2, \alpha)$ & $\downarrow_{6}$ & $\downarrow_{3}$ & $\downarrow_{2}$ & $\downarrow_{1}$ & $\uparrow_{1}$ & $\uparrow_{2}$ & $\uparrow_{4}$ & $\uparrow_{7}$ \\
$S(3, \alpha)$ & 0.791 & 0.893 & 0.946 & 0.978 & 1.022 & 1.056 & 1.113 & 1.231 \\
$\hat{S}(3, \alpha)$ & $\sim$ & $\sim$ & $\sim$ & $\sim$ & $\sim$ & $\sim$ & $\sim$ & $\sim$ \\
$\tilde{S}(3, \alpha)$ & $\uparrow_{19}$ & $\uparrow_{10}$ & $\uparrow_{5}$ & $\uparrow_{2}$ & $\downarrow_{2}$ & $\downarrow_{5}$ & $\downarrow_{11}$ & $\downarrow_{21}$ \\
$S(4, \alpha)$ & 0.808 & 0.904 & 0.952 & 0.981 & 1.019 & 1.049 & 1.097 & 1.196 \\
$\hat{S}(4, \alpha)$ & $\downarrow_{1}$ & $\downarrow_{1}$ & $\downarrow_{1}$ & $\sim$ & $\uparrow_{1}$ & $\sim$ & $\uparrow_{1}$ & $\uparrow_{1}$ \\
$\tilde{S}(4, \alpha)$ & $\uparrow_{11}$ & $\uparrow_{5}$ & $\uparrow_{2}$ & $\uparrow_{1}$ & $\downarrow_{1}$ & $\downarrow_{3}$ & $\downarrow_{5}$ & $\downarrow_{12}$ \\
\hline
\end{tabular}

Comparison of rainfall-runoff models in the Murray-Darling Basin

J. M. Whyte et al.

\section{Title Page}

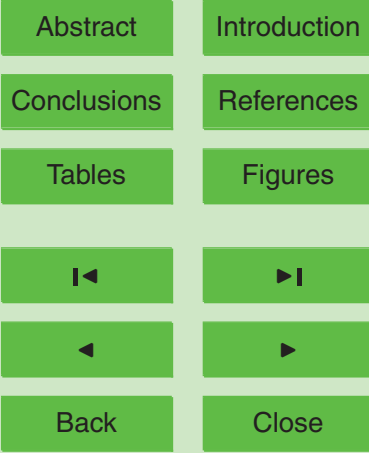

Full Screen / Esc

Printer-friendly Version

Interactive Discussion 


\section{HESSD}

8, 917-955, 2011

Table 9. Runoff scalings determined from deterministic and stochastic simulations using Model 5 for the four catchments considered with a range of values for rainfall scaling factor $\alpha$. All values are rounded to three decimal points. Symbols used are as for Table 7 .

\begin{tabular}{c|cccccccc}
\hline Model 5 & \multicolumn{8}{|c}{ Rainfall scaling $\alpha$} \\
runoff scaling & 0.8 & 0.9 & 0.95 & 0.98 & 1.02 & 1.05 & 1.1 & 1.2 \\
\hline$\hat{S}(1, \alpha)$ & 0.826 & 0.911 & 0.955 & 0.982 & 1.018 & 1.046 & 1.092 & 1.188 \\
$\tilde{S}(1, \alpha)$ & $\uparrow_{14}$ & $\uparrow_{8}$ & $\uparrow_{4}$ & $\uparrow_{1}$ & $\downarrow_{1}$ & $\downarrow_{4}$ & $\downarrow_{7}$ & $\downarrow_{15}$ \\
$\hat{S}(2, \alpha)$ & 0.868 & 0.933 & 0.966 & 0.986 & 1.014 & 1.034 & 1.069 & 1.139 \\
$\tilde{S}(2, \alpha)$ & $\uparrow_{17}$ & $\uparrow_{9}$ & $\uparrow_{5}$ & $\uparrow_{2}$ & $\downarrow_{2}$ & $\downarrow_{4}$ & $\downarrow_{9}$ & $\downarrow_{17}$ \\
$\hat{S}(3, \alpha)$ & 0.770 & 0.882 & 0.940 & 0.976 & 1.025 & 1.062 & 1.126 & 1.259 \\
$\tilde{S}(3, \alpha)$ & $\uparrow_{9}$ & $\uparrow_{4}$ & $\uparrow_{2}$ & $\uparrow_{1}$ & $\downarrow_{1}$ & $\downarrow_{2}$ & $\downarrow_{5}$ & $\downarrow_{10}$ \\
$\hat{S}(4, \alpha)$ & 0.826 & 0.912 & 0.955 & 0.982 & 1.018 & 1.045 & 1.091 & 1.186 \\
$\tilde{S}(4, \alpha)$ & $\uparrow_{26}$ & $\uparrow_{13}$ & $\uparrow_{7}$ & $\uparrow_{3}$ & $\downarrow_{3}$ & $\downarrow_{6}$ & $\downarrow_{13}$ & $\downarrow_{25}$ \\
\hline
\end{tabular}

Comparison of rainfall-runoff models in the Murray-Darling Basin

J. M. Whyte et al.

Title Page

Abstract Introduction

Conclusions References

Tables Figures

14

4

Back

Printer-friendly Version

Interactive Discussion 


\section{HESSD}

8, 917-955, 2011

Runoff scalings determined from deterministic predictions for a range of rainfall scaling factors and four catchments

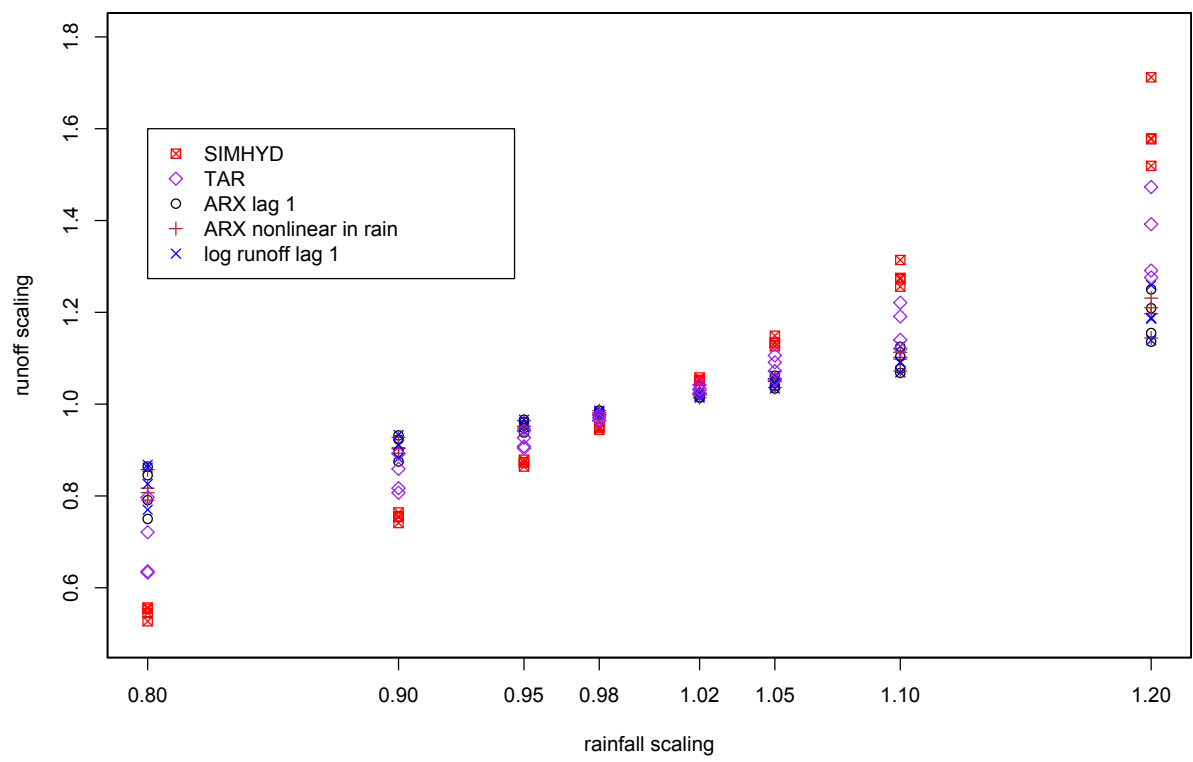

Fig. 1. Runoff scaling values determined from the deterministic predictions of SIMHYD, a two regime TARX model and Models 1, 3 and 5.

\section{Comparison of} rainfall-runoff models in the Murray-Darling Basin

J. M. Whyte et al.

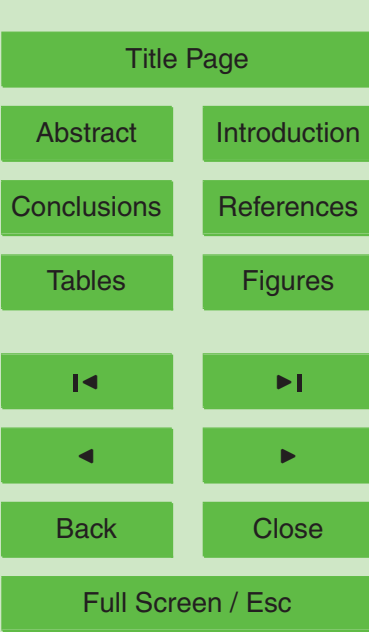

Printer-friendly Version

Interactive Discussion 